\title{
THE BLOCK STRUCTURE OF COMPLETE LATTICE ORDERED EFFECT ALGEBRAS
}

\author{
GEJZA JENČA \\ (Received 4 May 2005; revised 28 June 2006)
}

Communicated by M. Jackson

\begin{abstract}
We prove that every for every complete lattice-ordered effect algebra $E$ there exists an orthomodular lattice $O(E)$ and a surjective full morphism $\phi_{E}: O(E) \rightarrow E$ which preserves blocks in both directions: the (pre)image of a block is always a block. Moreover, there is a 0, 1-lattice embedding $\phi_{E}^{*}: E \rightarrow O(E)$.

2000 Mathematics subject classification: primary 06C15; secondary 03G12, 81P10.
\end{abstract}

\section{Introduction}

Effect algebras have recently been introduced by Foulis and Bennett in [9] for the study of the foundations of quantum mechanics. The class of effect algebras includes orthomodular lattices and a subclass equivalent to MV-algebras (see [4]).

In [30], Riečanová proved that every lattice-ordered effect algebra is a union of (essentially) MV-algebras. This result is a generalization of the well-known fact that every orthomodular lattice is a union of Boolean algebras. Generalizing the terminology from orthomodular lattices, a maximal sub-MV-effect algebra of a lattice-ordered effect algebra is called $a$ block. Later, Riečanová and Jenča proved in [24] that the set of all sharp elements of a lattice-ordered effect algebra forms an orthomodular lattice. Both papers show that the class of lattice-ordered effect algebras generalizes the class of orthomodular lattices in a very natural way. In [18] a new class, called homogeneous effect algebras was introduced and most of the results from [30]

This research is supported by grant VEGA G-1/3025/06 of MŠ SR. This work was supported by the Slovak Research and Development Agency under the contract No. APVV-0071-06.

(C) 2007 Australian Mathematical Society 1446-7887/07 \$A2.00+0.00 
and [24] were generalized for the homogeneous case. The main result of [18] is that every homogeneous effect algebra is a union of effect algebras satisfying the Riesz decomposition property.

Intuitively, one can consider the class of lattice-ordered effect algebras as an "unsharp" generalization of the class of orthomodular lattices, and the class of homogeneous effect algebras as an "unsharp" generalization of the class of orthoalgebras (see [10]). In these generalizations, the role of Boolean algebras is played by MV-effect algebras and by effect algebras with the Riesz decomposition property. The problems concerning this generalization were examined, for example, in [31] and [19]. The present paper continues this line of research.

The basic question we deal with in this paper is: "How are the blocks in a complete lattice-ordered effect algebra organized?". The main result is that for every complete lattice-ordered effect algebra $E$, there exists an orthomodular lattice $O(E)$ and a surjective full morphism of effect algebras $\phi_{E}: O(E) \rightarrow E$ such that for every block $B$ of $O(E), \phi_{E}(B)$ is a block and for every block $M$ of $E, \phi_{E}^{-1}(M)$ is a block of $O(E)$. This shows that the block structure of every complete lattice-ordered effect algebra is the same as the block structure of some orthomodular lattice. For the finite case, this result was proved in [19]. Moreover, we prove that the lattice $E$ can be 0,1 -embedded into the lattice $O(E)$.

Our construction of $O(E)$ is based on certain relations on the set of all quotients (that is, comparable pairs of elements) of $E$. We hope that it will be possible to adapt the techniques we have developed in this paper in order to deal with the more general orthocomplete non-lattice-ordered case. Most of the theorems were designed with this long-term goal in mind.

\section{Definition and basic relationships}

An effect algebra is a partial algebra $(E ; \oplus, 0,1)$ with a binary partial operation $\oplus$ and two nullary operations 0,1 satisfying the following conditions.

(E1) If $a \oplus b$ is defined then $b \oplus a$ is defined and $a \oplus b=b \oplus a$.

(E2) If $a \oplus b$ and $(a \oplus b) \oplus c$ are defined then $b \oplus c$ and $a \oplus(b \oplus c)$ are defined and $(a \oplus b) \oplus c=a \oplus(b \oplus c)$.

(E3) If $a \oplus b=a \oplus c$ then $b=c$.

(E4) If $a \oplus b=0$ then $a=0$.

(E5) For every $a \in E$ there is an $a^{\prime} \in E$ such that $a \oplus a^{\prime}=1$.

(E6) For every $a \in E, a \oplus 0=a$.

Effect algebras were introduced by Foulis and Bennett in their paper [9]. In the original paper, a different but equivalent set of axioms was used. 
In their paper [26], Chovanec and Kôpka introduced an essentially equivalent structure called a $D$-poset. Their definition is an abstract algebraic version the $D$-poset of fuzzy sets, introduced by Kôpka in the paper [25]. Another equivalent structure was introduced by Giuntini and Greuling in [12]. We refer the reader to [7] for more information on effect algebras and related topics.

One can construct examples of effect algebras from an arbitrary partially ordered abelian group $(G, \leq)$ in the following way: choose any positive $u \in G$; then, for $0 \leq a, b \leq u$, define $a \oplus b$ if and only if $a+b \leq u$ and put $a \oplus b=a+b$. With such a partial operation $\oplus$, the interval $[0, u]$ becomes an effect algebra $([0, u], \oplus, 0, u)$. Effect algebras which arise from partially ordered abelian groups in this way are called interval effect algebras, see [2].

In an effect algebra $E$, we write $a \leq b$ if and only if there is a $c \in E$ such that $a \oplus c=b$. It is easy to check that for every effect algebra the relation $\leq$ is a partial order on $E$. Moreover, it is possible to introduce a new partial operation $\ominus ; b \ominus a$ is defined if and only if $a \leq b$ and then $a \oplus(b \ominus a)=b$. It can be proved that, in an effect algebra, $a \oplus b$ is defined if and only if $a \leq b^{\prime}$, if and only if $b \leq a^{\prime}$. The partial operations $\oplus$ and $\Theta$ are connected by the rules

$$
\begin{aligned}
& a \oplus b=\left(a^{\prime} \ominus b\right)^{\prime} \\
& a \ominus b=\left(a^{\prime} \oplus b\right)^{\prime} .
\end{aligned}
$$

Let $E_{0} \subseteq E$ be such that $1 \in E_{0}$ and, for all $a, b \in E_{0}$ with $a \geq b, a \ominus b \in E_{0}$. Since $a^{\prime}=1 \ominus a$ and $a \oplus b=\left(a^{\prime} \ominus b\right)^{\prime}, E_{0}$ is closed with respect to $\oplus$ and '. We then say that $\left(E_{0}, \oplus, 0,1\right)$ is a subeffect algebra of $E$. Another way to construct a substructure of an effect algebra $E$ is to restrict $\oplus$ to an interval $[0, a]$, where $a \in E$, letting $a$ act as the unit element. We denote such effect algebra by $[0, a]_{E}$.

EXAMPLE 1. Let $G$ be the set of all real functions, partially ordered in the usual way. Let $u$ be the constant function $u(x)=1$. Then the restriction of + from $G$ to the set $[0, u]$ gives rise to an effect algebra, which we denote by $[0,1]^{[0,1]}$. Note that $[0,1]^{[0,1]}$ is a complete distributive lattice.

Let $E_{1}, E_{2}$ be effect algebras. A map $\phi: E_{1} \rightarrow E_{2}$ is called a morphism of effect algebras if and only if it satisfies the following condition.

(M1) $\phi(1)=1$ and, for all $a, b \in E_{1}$, if $a \oplus b$ exists in $E_{1}$ then $\phi(a) \oplus \phi(b)$ exists in $E_{2}$ and $\phi(a \oplus b)=\phi(a) \oplus \phi(b)$.

Every morphism preserves ', $0, \leq$ and $\Theta$.

A morphism $\phi: E_{1} \rightarrow E_{2}$ of effect algebras is called full if and only if the following condition is satisfied.

(M2) If $\phi(a) \oplus \phi(b)$ exists in $E_{2}$ and $\phi(a) \oplus \phi(b) \in \phi\left(E_{1}\right)$ then there exist $a_{1}, b_{1} \in E_{1}$ such that $a_{1} \oplus b_{1}$ exists in $E_{1}$ and $\phi(a)=\phi\left(a_{1}\right)$ and $\phi(b)=\phi\left(b_{1}\right)$. 
A bijective and full morphism is called an isomorphism of effect algebras.

An ideal of an effect algebra $E$ is a subset $I$ of $E$ satisfying the condition

$$
a, b \in I \quad \text { and } \quad a \oplus b \text { exists } \Longleftrightarrow a \oplus b \in I .
$$

The set of all ideals of an effect algebra $E$ is denoted by $I(E) . I(E)$ is a complete lattice with respect to inclusion.

An element $c$ of an effect algebra is central (see [14]) if and only if $[0, c]$ is an ideal and, for every $x \in E$, there is a decomposition $x=x_{1} \oplus x_{2}$ such that $x_{1} \leq c$ and $x_{2} \leq c^{\prime}$. It can be shown that this decomposition is unique. The set $C(E)$ of all central elements of an effect algebra is called the centre of $E$. $C(E)$ is a Boolean algebra. For every central element $c$ of $E, E$ is isomorphic to $[0, c]_{E} \times\left[0, c^{\prime}\right]_{E}$. For every central element $c$ of $E$ and every element $a \in E, a \wedge c$ exists and the mapping $a \mapsto a \wedge c$ is a full morphism from $E$ onto $[0, c]_{E}$; in other words, $\left(a_{1} \oplus a_{2}\right) \wedge c=\left(a_{1} \wedge c\right) \oplus\left(a_{2} \wedge c\right)$ and $a=(a \wedge c) \oplus\left(a \wedge c^{\prime}\right)$.

If $E$ is an effect algebra such that $(E, \leq)$ is a lattice, then we say that $E$ is $a$ lattice ordered effect algebra. If $(E, \leq)$ is a complete lattice, then we say that $E$ is a complete lattice ordered effect algebra. An orthoalgebra $E$ (see [11]) is an effect algebra such that $a \leq a^{\prime}$ implies $a=0$. It is easy to check that an effect algebra $E$ is an orthoalgebra if and only if $a \wedge a^{\prime}=0$ for all $a \in E$.

EXAMPLE 2. Recall, that an orthomodular lattice is an algebra $\left(O ; \vee, \wedge,{ }^{\prime}, 0,1\right)$ such that $(O ; \vee, \wedge, 0,1)$ is a bounded lattice and $a \leq b$ if and only if $b^{\prime} \leq a^{\prime}, a^{\prime \prime}=a$, $a \wedge a^{\prime}=0,(a \vee b)^{\prime}=a^{\prime} \wedge b^{\prime}$, and the orthomodular law

$$
a \leq b \quad \Longrightarrow \quad b=a \vee\left(b \wedge a^{\prime}\right)
$$

is satisfied. Equip $O$ with a partial operation as follows: $a \oplus b$ is defined if and only if $a \leq b^{\prime}$ and then $a \oplus b=a \vee b$. Then $(O ; \oplus, 0,1)$ is an orthoalgebra. On the other hand, for every lattice-ordered orthoalgebra $(O ; \oplus, 0,1),\left(O ; \vee, \wedge,{ }^{\prime}, 0,1\right)$ is an orthomodular lattice. However, there exist non-lattice-ordered orthoalgebras.

EXAMPLE 3. An $M V$-algebra (see $[4,28])$ is a commutative semigroup $(M ; \oplus, \neg, 0)$ satisfying the identities $x \oplus 0=x, \neg \neg x=x, x \oplus \neg 0=\neg 0$ and

$$
x \oplus \neg(x \oplus \neg y)=y \oplus \neg(y \oplus \neg x) .
$$

There is a natural partial order in an MV-algebra, given by $y \leq x$ if and only if $x=x \oplus \neg(x \oplus \neg y)$. Every MV-algebra $(M ; \oplus, \neg, 0)$ can be considered as an effect algebra $(M ; \oplus, 0, \neg 0)$, when we restrict the operation $\oplus$ to the domain $\{(x, y): x \leq \neg y\}$. 
Every lattice-ordered effect algebra satisfies the de Morgan laws. More generally, for an interval $[0, a]$ and $x, y \in[0, a]$, we have

$$
\begin{aligned}
& a \ominus(x \vee y)=(a \ominus x) \wedge(a \ominus y) \\
& a \ominus(x \wedge y)=(a \ominus x) \vee(a \ominus y) .
\end{aligned}
$$

A substitution $a=b^{\prime}$ and an application of (2.2) now yields

$$
\begin{aligned}
& b \oplus(x \vee y)=(b \oplus x) \vee(b \oplus y) \\
& b \oplus(x \wedge y)=(b \oplus x) \wedge(b \oplus y),
\end{aligned}
$$

for all $x, y \leq b^{\prime}$.

Let $E$ be a lattice-ordered effect algebra. For a pair of elements $a, b \in E$, the following conditions are equivalent:

- $a \ominus(a \wedge b)=(a \vee b) \ominus b$.

- $a \ominus(a \wedge b) \leq b^{\prime}$.

- There are $a_{1}, a_{2}$ such that $a=a_{1} \leq a_{2}, a_{1} \leq b, a_{2} \leq b^{\prime}$.

- There are $a_{1}, c, b_{1}$ such that $a_{1} \oplus c \oplus b_{1}$ exists and $a=a_{1} \oplus c$ and $b=b_{1} \oplus c$.

If $a, b$ satisfy any (or, equivalently, all) of these conditions then we say that $a, b$ are compatible (in symbols $a \leftrightarrow b$ ). It is easy to check that $a \leq b$ or $a \leq b^{\prime}$ implies that $a \leftrightarrow b$. Moreover, $a \leftrightarrow b$ if and only if $a \leftrightarrow b^{\prime}$. We say that a subset $A \subseteq E$ is compatible if and only if we have $a \leftrightarrow b$ for all $a, b \in A$. If $M$ is a lattice ordered effect algebra such that $M$ is a compatible subset of $M$ then we say that $M$ is an $M V$ effect algebra. It was proved in [5] that there is a natural, one-to-one correspondence between MV-effect algebras and MV-algebras, as outlined in Example 3. Every MVeffect algebra is a distributive lattice. A lattice-ordered effect algebra is an MV-effect algebra if and only if, for all elements $a, b$,

$$
a \wedge b=0 \quad \Longrightarrow \quad a \leq b^{\prime},
$$

that means, the sum of every disjoint pair exists (see [1]). An orthoalgebra that is an MV-effect algebra is a Boolean algebra.

Let $L$ be a lattice. We say that $L_{0} \subseteq L$ is a full sublattice of $L$ if and only if

- for all $A \subseteq L_{0}$ such that $\bigvee A$ exists in $L, \bigvee A \in L_{0}$, and

- for all $A \subseteq L_{0}$ such that $\bigwedge A$ exists in $L, \bigwedge A \in L_{0}$.

Note that a full sublattice of a complete lattice is complete.

Let $E$ be a lattice-ordered effect algebra. A subeffect algebra of $E$ that is maximal with respect to the property of being an MV-effect algebra is called a block. According to [30], blocks coincide with maximal compatible subsets of $E$. Moreover, every block is a full sublattice of $E$, (see [24]). Since every singleton is a compatible set, a latticeordered effect algebra is a union of its blocks. 
If $L$ is a compatible sublattice of a lattice-ordered effect algebra, then there is a block $M \supseteq L$. Since $M$ is a distributive lattice, $L$ is distributive as well.

If $E$ is an orthomodular lattice then every block is a Boolean algebra. Thus, the fact that every lattice-ordered effect algebra is a union of its blocks is a generalization of the well-known fact that every orthomodular lattice is a union of Boolean algebras.

We say that an element $a$ of an effect algebra is sharp if and only if $a \wedge a^{\prime}=0$. We write $S(E)$ for the set of all sharp elements of an effect algebra $E$. An orthoalgebra can be characterized by $E=S(E)$. Every central element is sharp, hence $C(E) \subseteq S(E)$. In general, $S(E)$ is not closed with respect to $\oplus$, (see [15]). However, by [24], if $E$ is lattice ordered, then $S(E)$ is a subeffect algebra and a full sublattice of $E$. For a block $M$ of a lattice-ordered effect algebra, we have $S(E) \cap M=S(M)=C(M)$, (see [24]).

An effect algebra $E$ is called homogeneous if and only if, for all $u, v_{1}, v_{2} \in E$ such that $u \leq v_{1} \oplus v_{2} \leq u^{\prime}$, there are $u_{1}, u_{2}$ such that $u_{1} \leq v_{1}, u_{2} \leq v_{2}$, and $u=u_{1} \oplus u_{2}$. Homogeneous effect algebras were introduced in [18]. Every orthoalgebra and every lattice-ordered effect algebra is homogeneous. The set of all sharp elements of a homogeneous effect algebra is closed with respect to $\oplus$, hence it forms an orthoalgebra.

In [18], most of the results (concerning compatibility, blocks, and sharp elements) from [30] and [24] were generalized for the homogeneous case. The situation is more complicated here. In a homogeneous effect algebra, the blocks need not be latticeordered anymore (they only satisfy the Riesz decomposition property) and the notion of compatibility has to be generalized as well.

EXAMPLE 4. Let $B$ be a Boolean algebra with at least two elements. Let us equip $B \times B$ with a partial $\oplus$ operation as follows: $\left\langle x_{1}, x_{2}\right\rangle \oplus\left\langle y_{1}, y_{2}\right\rangle$ is defined if and only if $x_{1} \wedge y_{2}=x_{2} \wedge y_{1}=0$ and then

$$
\left\langle x_{1}, x_{2}\right\rangle \oplus\left\langle y_{1}, y_{2}\right\rangle=\left\langle x_{1} \vee y_{1} \vee\left(x_{2} \wedge y_{2}\right), x_{2} \vee y_{2} \vee\left(x_{1} \wedge y_{1}\right)\right\rangle .
$$

Then $(B \times B ; \oplus,\langle 0,0\rangle,\langle 1,1\rangle)$ is an effect algebra denoted by $D^{B}$. In $D^{B}$, we have $\left\langle x_{1}, x_{2}\right\rangle \leq\left\langle y_{1}, y_{2}\right\rangle$ if and only if $x_{1} \leq x_{2}$ and $y_{1} \leq y_{2}$. Thus, $D^{B}$ is the same lattice as the "ordinary" Boolean lattice $B \times B$. However, if $B$ has more than one element then $D^{B}$ is not an MV-effect algebra: we have $\langle 1,0\rangle \wedge\langle 0,1\rangle=0$ but $\langle 1,0\rangle \oplus\langle 0,1\rangle$ does not exist.

It is easy to check that

$$
\left\langle x_{1}, x_{2}\right\rangle^{\prime}=\left\langle x_{2}^{\prime}, x_{1}^{\prime}\right\rangle
$$

and that

$$
S\left(D^{B}\right)=\{\langle x, x\rangle: x \in B\} .
$$

Note that $S\left(D^{B}\right)$ is a Boolean algebra. This implies that $S\left(D^{B}\right)=C\left(D^{B}\right)$. Since, for $|B|>1$, there are unsharp elements in $D^{B}, D^{B}$ is not an orthomodular lattice. 


\section{Generalized test spaces}

In this section we present a slightly generalized version of the notion of test space, originally introduced by Foulis and Randall in [11] and [29]. Despite of its relative simplicity, the notion of test space (and its generalizations) is a very useful tool for construction of orthoalgebras and effect algebras. See for example [6], [8], or [19] for constructions that use test spaces.

Let $X$ be a nonempty set and let $\mathscr{N}, \mathscr{T}$ be subsets of $2^{X}$. We say that a triple $(X, \mathscr{T}, \mathscr{N})$ is a generalized test space if and only if the following conditions are satisfied.

(GTS1) $X=\cup_{\mathfrak{t} \in \mathscr{T}} \mathbf{t}$.

(GTS2) $\mathscr{N}$ is an ideal of $2^{X}$, that is, $\mathscr{N}$ is nonempty and for all $\mathbf{o}_{1}, \mathbf{o}_{2} \subseteq X$ we have $\mathbf{o}_{1} \cup \mathbf{o}_{2} \in \mathscr{N}$ if and only if $\mathbf{o}_{1}, \mathbf{o}_{2} \in \mathscr{N}$.

(GTS3) For all $\mathbf{t}_{1} \subseteq \mathbf{t}_{2} \subseteq X$ such that $\mathbf{t}_{1} \in \mathscr{T}$, we have $\mathbf{t}_{2} \in \mathscr{T}$ if and only if $\mathbf{t}_{2} \backslash \mathbf{t}_{1} \in \mathscr{N}$.

(GTS4) For all $\mathbf{t}_{1} \subseteq \mathbf{t}_{2} \subseteq X$ such that $\mathbf{t}_{2} \backslash \mathbf{t}_{1} \in \mathscr{N}$, we have $\mathbf{t}_{1} \in \mathscr{T}$ if and only if $\mathbf{t}_{2} \in \mathscr{T}$.

A generalized test space is a test space if and only if $\mathscr{N}=\{\emptyset\}$. For a test space, the Axioms (GTS2) and (GTS4) collapse to tautologies and (GTS3) transforms to

(TS) If $\mathbf{t}_{1}, \mathbf{t}_{2} \in \mathscr{T}$ and $\mathbf{t}_{1} \subseteq \mathbf{t}_{2}$, then $\mathbf{t}_{1}=\mathbf{t}_{2}$.

(GTS1) and (TS) are the original axioms of a test space.

EXAMPLE 5. Let $X$ be the system of all measurable subsets of the real interval $[0,1]_{\mathbb{R}}$ and let $\mathscr{T}$ be the set of all finite systems $\mathbf{t} \subseteq X$ such that the elements of $\mathbf{t}$ are measurable, pairwise disjoint and $\mu\left(\dot{\cup}_{A \in t} A\right)=1$. Let $\mathscr{N}$ be the set of all finite pairwise disjoint systems of sets with zero measure. Then $(X, \mathscr{T}, \mathscr{N})$ is a generalized test space.

Throughout this section, we assume that $(X, \mathscr{T}, \mathscr{N})$ is a generalized test space.

An element of $\mathscr{T}$ is called a test. Since $X$ is nonempty, (GTS1) implies that there is at least one test. We say that a subset $f$ of $X$ is an event if and only if there is a test $\mathbf{t} \in \mathscr{T}$ such that $\mathbf{f} \subseteq \mathbf{t}$.

LEMMA 3.1. Every element of $\mathscr{N}$ is an event.

Proof. Let $\mathbf{t}_{1}$ be a test and let $\mathbf{o} \in \mathscr{N}$. Put $\mathbf{t}_{2}=\mathbf{t}_{1} \cup \mathbf{0}$. We have $\mathbf{t}_{2} \backslash \mathbf{t}_{1} \subseteq \mathbf{o} \in \mathscr{N}$. Since $\mathscr{N}$ is an ideal of sets, $\mathbf{t}_{2} \backslash \mathbf{t}_{1} \in \mathscr{N}$. By (GTS3), $\mathbf{t}_{1} \in \mathscr{T}$ and $\mathbf{t}_{2} \backslash \mathbf{t}_{1} \in \mathscr{N}$ imply that $\mathbf{t}_{2}$ is a test. Thus, $\mathbf{0} \subseteq \mathbf{t}_{\mathbf{2}}$ is an event.

The elements of $\mathscr{N}$ are called null events. 
We say that two events $\mathbf{f}, \mathbf{g}$ of a generalized test space $(X, \mathscr{T}, \mathscr{N})$ are

(i) orthogonal (in symbols $\mathbf{f} \perp \mathbf{g}$ ) if and only if $\mathbf{f} \cap \mathbf{g} \in \mathscr{N}$ and $\mathbf{f} \cup \mathbf{g}$ is an event.

(ii) local complements (in symbols $\mathbf{f}$ loc $\mathbf{g}$ ) if and only if $\mathbf{f} \cap \mathbf{g} \in \mathscr{N}$ and $\mathbf{f} \cup \mathbf{g}$ is a test.

(iii) perspective (in symbols $\mathbf{f} \sim \mathbf{g}$ ) if and only if they share a local complement.

Note that every pair of tests is perspective, since $\emptyset$ is a local complement of every test.

LEMMA 3.2. For an event $\mathbf{f}$ we have $\mathbf{f} \sim \emptyset$ if and only if $\mathbf{f} \in \mathscr{N}$.

PROOF. Suppose that $\mathbf{f} \sim \emptyset$. There is a test $\mathbf{t}$ such that $\mathbf{f}$ loc $\mathbf{t}$. Since $\mathbf{f} \cup \mathbf{t}$ is a test, (GTS3) implies that $\mathbf{f} \cup \mathbf{t} \backslash \mathbf{t}=\mathbf{f} \backslash \mathbf{f} \cap \mathbf{t}$ is a null event. Since $\mathbf{f}$ loc $\mathbf{t}, \mathbf{f} \cap \mathbf{t}$ is a null event. Therefore, $f=(f \backslash f \cap t) \cup(f \cap t)$ is a null event.

Suppose that $\mathbf{f} \in \mathscr{N}$. Since $\mathbf{f}$ is an event, there is a test $\mathbf{t} \supseteq \mathbf{f}$. Since $\mathbf{t} \cap \mathbf{f}=\mathbf{f} \in \mathscr{N}$ and $\mathbf{t} \cup \mathbf{f}=\mathbf{t} \in \mathscr{T}, \mathbf{f}$ loc $\mathbf{t}$. Since $\mathbf{t}$ is a test, $\emptyset$ loc $\mathbf{t}$. Therefore, $\mathbf{f} \sim \emptyset$.

LEMMA 3.3. Let $\mathbf{t}$ be a test and let $\mathbf{f}$ be an event such that $\mathbf{f} \sim \mathbf{t}$. Then $\mathbf{f}$ is a test.

PROOF. Let $h$ be a local complement shared by $f$ and $t$. Both $t \cup h$ and $t$ are tests. By (GTS3), $\mathbf{t} \cup \mathbf{h} \backslash \mathbf{t} \in \mathscr{N}$. Since $\mathbf{t} \mathbf{l o c} \mathbf{h}, \mathbf{t} \cap \mathbf{h} \in \mathscr{N}$. Therefore, $\mathbf{h}=(\mathbf{t} \cup \mathbf{h} \backslash \mathbf{t}) \cup(\mathbf{t} \cap \mathbf{h}) \in \mathscr{N}$ and, since $\mathbf{f} \cup \mathbf{h} \backslash \mathbf{f} \subseteq \mathbf{h}, \mathbf{f} \cup \mathbf{h} \backslash \mathbf{f} \in \mathscr{N}$. By (GTS4), $\mathbf{f} \cup \mathbf{h} \in \mathscr{T}$ and $\mathbf{f} \cup \mathbf{h} \backslash \mathbf{f} \in \mathscr{N}$ imply that $\mathbf{f} \in \mathscr{T}$.

We say that a generalized test space is algebraic if and only if for all events $\mathbf{f}, \mathbf{g}, \mathbf{h}$ we have

$$
\mathbf{f} \sim \mathbf{g} \quad \text { and } \quad \mathbf{f l o c} \mathbf{h} \quad \Longrightarrow \quad \mathbf{g l o c} \mathbf{h} .
$$

LEMMA 3.4. In an algebraic generalized test space, perspectivity is transitive.

Proof. Suppose that $\mathbf{f} \sim \mathbf{g} \sim \mathbf{h}$. There are events $\mathbf{u}_{1}$, $\mathbf{u}_{2}$ such that $\mathbf{f}$ loc $\mathbf{u}_{1} \operatorname{loc} \mathbf{g}$ and $\mathbf{g} \operatorname{loc} \mathbf{u}_{2} \operatorname{loc} \mathbf{h}$. Since $\mathbf{u}_{2} \operatorname{loc} \mathbf{h}$ and $\mathbf{u}_{1} \sim \mathbf{u}_{2}, \mathbf{u}_{1}$ loc $\mathbf{h}$. Therefore, $\mathbf{f} \sim \mathbf{h}$.

Note that, in an algebraic generalized test space, both $\mathscr{N}$ and $\mathscr{T}$ are equivalence classes of $\sim$.

LEMMA 3.5. In an algebraic generalized test space, if $\mathbf{f}_{1} \sim \mathbf{f}_{2}$ and $\mathbf{f}_{1} \perp \mathbf{g}$ then $\mathbf{f}_{2} \perp \mathbf{g}$ and $\mathbf{f}_{1} \cup \mathbf{g} \sim \mathbf{f}_{2} \cup \mathbf{g}$.

PROOF. Let $h$ be a local complement of $f_{1} \cup \mathbf{g}$. Since $f_{1} \operatorname{loc} h \cup g$ and $f_{1} \sim f_{2}$, $\mathbf{f}_{2} \operatorname{loc} \mathbf{h} \cup \mathbf{g}$. This implies that $\mathbf{f}_{2} \perp$ g. Moreover, as $\mathbf{f}_{\mathbf{1}} \cup \mathbf{g} \operatorname{loc} \mathbf{h} \operatorname{loc} \mathbf{f}_{2} \cup \mathbf{g}$, $\mathbf{f}_{1} \cup \mathbf{g} \sim \mathbf{f}_{2} \cup \mathbf{g}$. 
THEOREM 3.6. Let $(X, \mathscr{T}, \mathscr{N})$ be an algebraic generalized test space. Let $\mathscr{E}$ be the set of all events of $(X, \mathscr{T}, \mathscr{N})$. Define on $\mathscr{E} / \sim$ a relation $\perp$ and a partial operation $\oplus$ with domain $\perp$ in the following way: $[\mathbf{f}] \sim \perp$ [g] if and only if $\mathbf{f} \perp \mathbf{g}$ and then $[\mathbf{f}]_{\sim} \oplus[\mathbf{g}]_{\sim}=[\mathbf{f} \cup \mathbf{g}]_{\sim}$. Then $(\mathscr{E} / \sim, \oplus, \mathscr{N}, \mathscr{T})$ is an orthoalgebra.

Proof. Let us prove that $\perp$ and $\oplus$ are well-defined. Suppose that $\mathbf{f}_{1} \sim \mathbf{f}_{2}$ and $\mathbf{g}_{1} \sim \mathbf{g}_{2}$ and $\mathbf{f}_{1} \perp \mathbf{g}_{1}$. By Lemma 3.5, $\mathbf{f}_{1} \perp \mathbf{g}_{2}$ and $\mathbf{f}_{1} \cup \mathbf{g}_{1} \sim \mathbf{f}_{1} \cup \mathbf{g}_{2}$. Again, by Lemma 3.5, this implies that $\mathbf{f}_{2} \perp \mathbf{g}_{2}$ and $\mathbf{f}_{1} \cup \mathbf{g}_{2} \sim \mathbf{f}_{2} \cup \mathbf{g}_{2}$.

(E1) is trivially true, so let us prove (E2). If both sides of the associative equality exist, they are (obviously) equal. Suppose that $\left([\mathbf{f}]_{\sim} \oplus[\mathbf{g}]_{\sim}\right) \oplus[\mathbf{h}]_{\sim}$ exists. Then $\mathbf{f} \perp \mathbf{g}$ and $\left([\mathbf{f}]_{\sim} \oplus[\mathbf{g}]_{\sim}\right)=[\mathbf{f} \cup \mathbf{g}]_{\sim}$. Therefore $\mathbf{f} \cup \mathbf{g} \perp \mathbf{h}$ and we see that $[\mathbf{f}]_{\sim} \oplus\left([\mathbf{g}]_{\sim} \oplus[\mathbf{h}]_{\sim}\right)$ exists.

Let us prove (E3). Suppose that $[\mathbf{f}]_{\sim} \oplus[\mathbf{h}]_{\sim}=[\mathbf{g}]_{\sim} \oplus[\mathbf{h}]_{\sim}$, that means, $\mathbf{f}, \mathbf{g} \perp \mathbf{h}$ and $\mathbf{f} \cup \mathbf{h} \sim \mathbf{g} \cup \mathbf{h}$; let $\mathbf{u}$ be a common local complement of $\mathbf{f} \cup \mathbf{h}$ and $\mathbf{g} \cup \mathbf{h}$. We see that $\mathbf{h} \cup \mathbf{u}$ is a common local complement of $\mathbf{f}$ and $\mathbf{g}$, therefore $\mathbf{f} \sim \mathbf{g}$ and $[\mathbf{f}]_{\sim}=[\mathrm{g}]_{\sim}$.

(E4) is trivial, so let us prove (E5). For an event $\mathbf{f},[\mathbf{f}]_{\sim}^{\prime}$ is just the set of all local complements of $\mathbf{f}$. Obviously, [f] $]_{\sim}^{\prime}$ can be characterized by the property $[\mathbf{f}]_{\sim} \oplus[\mathbf{f}]_{\sim}^{\prime}=\mathscr{T}$.

The proof of (E6) is trivial.

Finally, let us prove that $[\mathbf{f}]_{\sim} \leq[\mathbf{f}]_{\sim}^{\prime}$ implies $[\mathbf{f}]_{\sim}=\mathscr{N}$. Since $\mathbf{f} \perp \mathbf{f}$, we have $\mathbf{f} \cap \mathbf{f}=\mathbf{f} \in \mathscr{N}$. Therefore [f] $]_{\sim}=\mathscr{N}$ and we see that $(\mathscr{E} / \sim, \oplus, \mathscr{N}, \mathscr{T})$ is an orthoalgebra.

The orthoalgebra $(\mathscr{E} / \sim, \oplus, \mathscr{N}, \mathscr{T})$ is called the orthoalgebra of the generalized test space $(X, \mathscr{T}, \mathscr{N})$.

\section{The generalized test space of quotients}

In this section we introduce our main tool. For every homogeneous effect algebra $E$ we shall construct a generalized test space $\Omega(E)$, where tests are finite sets of comparable pairs (called quotients) with certain properties.

The origins of the notion of a quotient and the relations $\nearrow$ and $\searrow$ lie in lattice theory, see for example [13, Section III.1]. However, the definitions of $\nearrow$ and $\searrow$ introduced here do not coincide with their lattice-theoretical versions, even in the case of a lattice-ordered effect algebra (see the remark following Proposition 4.3). In the case of an MV-effect algebra, our definitions coincide with their lattice-theoretical counterparts.

Let $E$ be an effect algebra and let $P$ be a subposet of $E$. Let $a / b$ denote an ordered pair of elements of $P$ satisfying $a \geq b$. We say that $a / b$ is a quotient of $P$. The set 
of all quotients of $P$ is denoted by $Q(P)$. We say that $c / d$ is a subquotient of $a / b$ (in symbols $c / d \sqsubseteq a / b$ or $a / b \sqsupseteq c / d)$ if and only if $b \leq d \leq c \leq a$.

If $a>b$, we say that $a / b$ is proper, otherwise we say that $a / b$ is null.

We write $a / b \nearrow c / d$ if and only if $a \leq c, b \leq d, c \ominus a=d \ominus b$ and $(c \ominus a) \wedge(a \ominus b)=0$. We write $a / b \searrow c / d$ if and only if $c \leq a, d \leq b$, $a \ominus c=b \ominus d$ and $(a \ominus c) \wedge(a \ominus b)=0$. It is easy to check that $a / b \searrow c / d$ or $a / b \nearrow c / d$ implies that $a \ominus b=c \ominus d$.

PROPOSITION 4.1. Let $E$ be an effect algebra and let $a / b, c / d \in Q(E)$. The following are equivalent:

(i) $a / b \searrow c / d$;

(ii) $c / d \nearrow a / b$;

(iii) $b^{\prime} / a^{\prime} \nearrow d^{\prime} / c^{\prime}$;

(iv) $d^{\prime} / c^{\prime} \searrow b^{\prime} / a^{\prime}$.

PROOF. The proofs of the equivalence of (i) and (ii) and of (iii) and (iv) are trivial.

Let us prove that (i) implies (iii). Since $c \leq a, a^{\prime} \leq c^{\prime}$. Since $d \leq b, b^{\prime} \leq d^{\prime}$. We have

$$
d^{\prime} \ominus b^{\prime}=\left(d^{\prime} \ominus b^{\prime}\right)^{\prime \prime}=\left(d \oplus b^{\prime}\right)^{\prime}=\left(b^{\prime} \oplus d\right)^{\prime}=b \ominus d .
$$

Similarly, $c^{\prime} \ominus a^{\prime}=a \ominus c$ and $b^{\prime} \ominus a^{\prime}=a \ominus b$. Therefore,

$$
d^{\prime} \ominus b^{\prime}=b \ominus d=a \ominus c=c^{\prime} \ominus a^{\prime}
$$

and

$$
\left(d^{\prime} \ominus b^{\prime}\right) \wedge\left(b^{\prime} \ominus a^{\prime}\right)=(b \ominus d) \wedge(a \ominus b)=(a \ominus c) \wedge(a \ominus b)=0 .
$$

Let us prove that (iii) implies (i). By the previous parts of the proof,

$$
b^{\prime} / a^{\prime} \nearrow d^{\prime} / c^{\prime} \Longrightarrow d^{\prime} / c^{\prime} \searrow b^{\prime} / a^{\prime} \Longrightarrow c / d \nearrow a / b \Longrightarrow a / b \searrow c / d \text {. }
$$

EXAMPLE 6. Let $[0,1]^{[0,1]}$ be the effect algebra of all real functions of a real variable $[0,1] \rightarrow[0,1]$. For $a / b, c / d \in Q\left([0,1]^{[0,1]}\right)$ we have $a / b \searrow c / d$ if and only if, for all $x \in[0,1], a(x) \neq c(x)$ or $b(x) \neq d(x)$ imply $a(x)=b(x) \geq c(x)=d(x)$.

For example, we may take

$$
\begin{array}{ll}
a(x)=|x-0.5|+0.5, & c(x)=1-x, \\
b(x)=x, & d(x)=0.5-|x-0.5|
\end{array}
$$

(see Figure 1).

EXAMPLE 7. Let $E$ be a 6-element effect algebra with two atoms $a, b$, satisfying $a \oplus a \oplus a=a \oplus b \oplus b=1$. On $Q(E)$, the $\searrow$ relation is not transitive: we have $1 / a^{\prime} \searrow a \oplus b / b$ and $a \oplus b / b \searrow a / 0$, but $1 / a^{\prime} \backslash a / 0$, because $a \wedge a^{\prime}=a \neq 0$. 

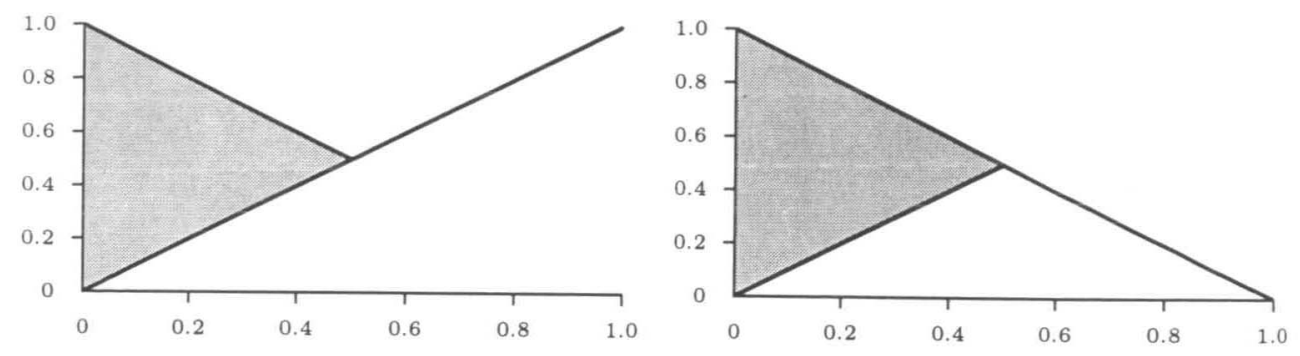

FIGURE $1 . a / b \searrow c / d$ in $[0,1]^{[0,1]}$

PROPOSITION 4.2. For every homogeneous effect algebra, $\nearrow$ and $\searrow$ are transitive.

PROOF. Let $E$ be a homogeneous effect algebra and let $a / b, c / d, e / f \in Q(E)$. Assume that $a / b \nearrow c / d$ and $c / d \nearrow e / f$. Obviously $a \leq e$ and $b \leq f$. We see that

$$
e \ominus a=(e \ominus c) \oplus(c \ominus a)=(f \ominus d) \oplus(d \ominus b)=f \ominus b .
$$

Suppose that $x \leq e \ominus a, a \ominus b$. Since $e \ominus a \leq(a \ominus b)^{\prime} \leq x^{\prime}$ and $e \ominus a=(e \ominus c) \oplus(c \ominus a)$, we obtain

$$
x \leq(e \ominus c) \oplus(c \ominus a) \leq x^{\prime} .
$$

Since $E$ is homogeneous, there exist $x_{1} \leq e \ominus c$ and $x_{2} \leq c \ominus a$ such that $x=x_{1} \oplus x_{2}$. However, as $c \ominus d=a \ominus b$, we have $(e \ominus c) \wedge(c \ominus d)=(e \ominus c) \wedge(a \ominus b)=0$ and thus $x_{1} \leq e \ominus c, a \ominus b$ implies that $x_{1}=0$. As $x_{2} \leq c \ominus a, a \ominus b$ and $(c \ominus a) \wedge(a \ominus b)=0$, $x_{2}=0$. Therefore, $(e \ominus a) \wedge(a \ominus b)=0$ and $a / b \nearrow e / f$.

Assume that $a / b \searrow c / d$ and $c / d \searrow e / f$. By Proposition 4.1, this is equivalent to $b^{\prime} / a^{\prime} \nearrow d^{\prime} / c^{\prime}$ and $d^{\prime} / c^{\prime} \nearrow f^{\prime} / e^{\prime}$. Since $\nearrow$ is transitive, $b^{\prime} / a^{\prime} \nearrow f^{\prime} / e^{\prime}$ and hence $a / b \searrow e / f$.

PROPOSITION 4.3. Let $E$ be a lattice-ordered effect algebra and let $a / b, c / d \in$ $Q(E)$. Then

(i) $a / b \nearrow c / d$ if and only if $a \leftrightarrow d, a \vee d=c, a \wedge d=b$,

(ii) $a / b \searrow c / d$ if and only if $c \leftrightarrow b, c \vee b=a, c \wedge b=d$.

PROOF. (i) Suppose that $a / b \nearrow c / d$. Since $(c \ominus a) \oplus(a \ominus b) \oplus b$ exists, the set $\{c \ominus a, a \ominus b, b\}$ is compatible and can be embedded into a block $M$. As $a=(a \ominus b) \oplus b$, $a \in M$. Since $c \ominus a=d \ominus b$, we see that $d=(d \ominus b) \oplus b=(c \ominus a) \oplus b \in M$. Therefore, $a \leftrightarrow d$. We see that

$$
c \ominus(a \vee d)=(c \ominus a) \wedge(c \ominus d)=(d \ominus b) \wedge(a \ominus b)=0 .
$$

Therefore $c=a \vee d$. 
Since $a \leftrightarrow d$ and $c \ominus d=a \ominus b$,

$$
c \ominus d=(a \vee d) \ominus d=a \ominus(a \wedge d)=a \ominus b .
$$

Therefore $a \wedge d=b$.

Suppose that $a \leftrightarrow d, a \vee d=c$ and $a \wedge d=b$. Obviously, $a \wedge d \leq a$ and $d \leq a \vee d$. Since $a \leftrightarrow d,(a \vee d) \ominus a=d \ominus(a \wedge b)$ and it is easy to check that $[a \ominus(a \wedge d)] \wedge[d \ominus(a \wedge d)]=0$.

(ii) This follows from (i) by a permutation of $\{a, b, c, d\}$.

REMARK. In lattice theory, the relation $\nearrow$ is defined by the rule

$$
a / b \nearrow c / d \quad \Longleftrightarrow a \vee d=c \quad \text { and } a \wedge d=b
$$

and $\searrow$ is defined in a dual way. By Proposition 4.3, our "effect-algebraic $\nearrow$ " is more restrictive than the original lattice-theoretical $\nearrow$. Both definitions coincide for MV-effect algebras, because in this case the additional condition $a \leftrightarrow d$ is clearly satisfied.

In what follows, the symbol $\equiv$ denotes the transitive closure of $(\searrow \cup \nearrow)$. Obviously, $\equiv$ is an equivalence relation.

EXAMPLE 8. Let $a / b, c / d \in Q\left([0,1]^{[0,1]}\right)$. We have $a / b \equiv c / d$ if and only if for all $x \in[0,1]$

$$
a(x) \neq c(x) \quad \text { or } \quad b(x) \neq d(x) \quad \Longrightarrow \quad a(x)=b(x) \quad \text { and } \quad c(x)=d(x) .
$$

We say that quotients $a / b$ and $c / d$ are disjoint if and only if for all $x / y$ and $z / w$,

$$
a / b \sqsupseteq x / y \equiv z / w \sqsubseteq c / d \quad \Longrightarrow \quad x=y .
$$

We say that $a / b$ and $c / d$ are orthogonal (in symbols $a / b \perp c / d$ ) if and only if $a / b$ and $c / d$ are disjoint and $(a \ominus b) \oplus(c \ominus d)$ exists in $E$. We say that a finite set $\mathbf{f}$ of quotients is pairwise orthogonal if and only if any two distinct elements of $\mathbf{f}$ are orthogonal. We say that a finite set of quotients $\mathbf{f}=\left\{a_{1} / b_{1}, \ldots, a_{n} / b_{n}\right\}$ is orthogonal if and only if $\mathbf{f}$ is pairwise orthogonal and the sum $|\mathbf{f}|$ defined by

$$
|\mathbf{f}|=\left(a_{1} \ominus b_{1}\right) \oplus \cdots \oplus\left(a_{n} \ominus b_{n}\right)
$$

exists in $E$.

EXAMPLE 9. In $[0,1]^{[0,1]}$, we have $a / b \perp c / d$ if and only if, for all $x \in[0,1]$, the intervals $(b(x), a(x)]$ and $(d(x), c(x)]$ are disjoint. 


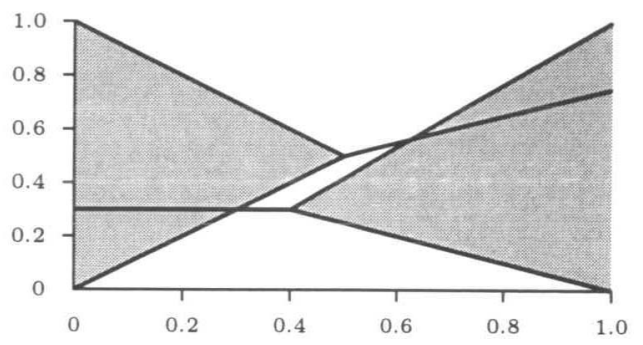

FIGURE 2. $a / b \perp c / d$ in $[0,1]^{[0,1]}$

EXAMPLE 10. In an orthoalgebra, we have $a / b \perp c / d$ if and only if $(a \Theta b) \oplus(c \Theta d)$ exists.

Note that, for $a / b \nearrow c / d$ and $x / y \sqsubseteq a / b$, there is $x_{0} / y_{0} \sqsubseteq c / d$ with $x / y \nearrow x_{0} / y_{0}$. Indeed, we may put $x_{0}=x \oplus(c \ominus a)$ and $y_{0}=y \oplus(c \ominus a)$. There is an analogous relationship between $\searrow$ and $\sqsubseteq$.

PROPOSITION 4.4. Let $E$ be a homogeneous effect algebra and let $a / b, c / d, e / f \in$ $Q(E)$. If $a / b \equiv c / d$ and $c / d$ is disjoint with $e / f$, then $a / b$ is disjoint with $e / f$.

PROOF. Suppose that $a / b \nearrow c / d$. Let $x / y$ and $z / w$ be such that

$$
a / b \sqsupseteq x / y \equiv z / w \sqsubseteq e / f \text {. }
$$

There is $x_{0} / y_{0} \sqsubseteq c / d$ with $x / y \nearrow x_{0} / y_{0}$. However, since $c / d \sqsupseteq x_{0} / y_{0} \equiv z / w \sqsubseteq e / f$ and $c / d, e / f$ are disjoint, $x_{0}=y_{0}$ and hence $x=y$.

Similarly, $a / b \searrow c / d$ implies that $a / b$ is disjoint with $e / f$. The rest of the proof is a simple induction.

Let $E$ be a homogeneous effect algebra. Let us extend the relation $\equiv$ to the set of all finite subsets of $Q(E)$ : for two finite sets of quotients $\mathbf{f}$ and $\mathbf{g}$ we write $\mathbf{f} \equiv \mathbf{g}$ if and only if the following symmetric pair of conditions is satisfied.

- For every proper $a / b \in \mathbf{f}$ there is exactly one $c / d \in \mathbf{g}$ such that $a / b \equiv c / d$.

- For every proper $a / b \in \mathbf{g}$ there is exactly one $c / d \in \mathbf{f}$ such that $a / b \equiv c / d$.

It is obvious that $\equiv$ is an equivalence relation on the set of all finite sets of quotients. Note that $\mathbf{f} \equiv \emptyset$ if and only if $\mathbf{f}$ contains only null quotients.

LEMMA 4.5. Let $E$ be a homogeneous effect algebra and let $\mathbf{f}, \mathbf{g}$ be finite sets of quotients. If $\mathbf{f} \equiv \mathbf{g}$ and $\mathbf{f}$ is (pairwise) orthogonal, then $\mathbf{g}$ is (pairwise) orthogonal.

PROOF. Suppose that $\mathbf{f}$ is pairwise orthogonal. Let $a_{1} / b_{1}, a_{2} / b_{2} \in \mathbf{g}$ such that $a_{1} / b_{1} \neq a_{2} / b_{2}$. If one of $a_{1} / b_{1}, a_{2} / b_{2}$ is null then $a_{1} / b_{1} \perp a_{2} / b_{2}$, so let us assume 
that both $a_{1} / b_{1}, a_{2} / b_{2}$ are proper. Since $\mathbf{f} \equiv \mathbf{g}$, there are $c_{1} / d_{1}, c_{2} / d_{2} \in \mathbf{f}$ such that $c_{1} / d_{1} \equiv a_{1} / b_{1}, c_{2} / d_{2} \equiv a_{2} / b_{2}$ and $c_{1} / d_{1} \neq c_{2} / d_{2}$. Since $\mathbf{f}$ is pairwise orthogonal, $c_{1} / d_{1} \perp c_{2} / d_{2}$. Therefore, $a_{1} / b_{1} \perp a_{2} / b_{2}$.

Suppose that $\mathbf{f}$ is orthogonal. Then $\mathbf{g}$ is pairwise orthogonal and it remains to observe that the elements occurring in the sum $|\mathbf{f}|$ are (up to some zeros) the same as the elements occurring in the sum $|\mathbf{g}|$. Therefore, $\mathbf{g}$ is orthogonal.

LEMMA 4.6. Let $E$ be a homogeneous effect algebra and let

$$
\mathbf{f}=\left\{a_{1} / b_{1}, \ldots, a_{n} / b_{n}\right\} \subseteq Q(E)
$$

be a pairwise orthogonal $n$-element set. Let

$$
\mathbf{g}=\left\{c_{1} / d_{1}, \ldots, c_{n} / d_{n}\right\} \subseteq Q(E)
$$

be a finite set such that, for all $i \in\{1, \ldots, n\}, c_{i} / d_{i} \equiv a_{i} / b_{i}$. Then $\mathbf{f} \equiv \mathbf{g}$.

PROOF. We have to prove that, for proper $a_{j} / b_{j}$ and $c_{i} / d_{i}, a_{j} / b_{j} \equiv c_{i} / d_{i}$ implies that $i=j$. Suppose that $i \neq j$. Since $\mathbf{f}$ is pairwise orthogonal, $a_{i} / b_{i} \perp a_{j} / b_{j}$. However, $a_{i} / b_{i} \equiv c_{i} / d_{i} \equiv a_{j} / b_{j}$. Therefore, $a_{i} / b_{i} \equiv a_{j} / b_{j}$ and this is a contradiction with $a_{i} / b_{i} \perp a_{j} / b_{j}$.

Note that we cannot omit the assumption that $\mathbf{f}$ is pairwise orthogonal from Lemma 4.6. To see this, let $\mathbf{f}=\left\{a_{1} / b_{1}, a_{2} / b_{2}\right\}$ be such that $a_{1} / b_{1} \equiv a_{2} / b_{2}$ and $a_{1} / b_{1}$ is proper. Then for $\mathbf{g}=\left\{a_{1} / b_{1}, a_{1} / b_{1}\right\}$ we have $\mathbf{g} \not \equiv \mathbf{f}$.

Let $E$ be a homogeneous effect algebra. Let $\mathscr{T}$ be the set of all finite orthogonal sets $\mathbf{t} \subseteq Q(E)$ with $|\mathbf{t}|=1$ and let $\mathscr{N}$ be the set of all finite sets of null quotients. We define a triple $\Omega(E)$ by $\Omega(E)=(Q(E), \mathscr{T}, \mathscr{N})$. It is evident that $\Omega(E)$ forms a generalized test space. Note that, for all events $\mathbf{f}, \mathbf{g}$ of $\Omega(E), \mathbf{f} \equiv \mathbf{g}$ implies that $\mathbf{f} \sim \mathbf{g}$.

The main aim of the following two sections of this paper is to prove the following theorem.

THEOREM 4.7. Let $E$ be a complete lattice-ordered effect algebra. Let $\mathbf{f}$ be a finite set of quotients of $E$. Then the following are equivalent:

(a) $\mathbf{f}$ is an event of $\Omega(E)$;

(b) $\mathbf{f}$ is an orthogonal set of quotients;

(c) $\mathbf{f}$ is a pairwise orthogonal set of quotients.

It will then turn out that $\Omega(E)$ is an algebraic generalized test space. Later we shall prove that the orthoalgebra $O(E)$ of $\Omega(E)$ is actually an orthomodular lattice with the same block structure as $E$. 


\section{Reduced quotients}

Let $E$ be an effect algebra and let $a / b \in Q(E)$. We say that $a / b$ is a reduced quotient if and only if

$$
a / b \searrow c / d \quad \Longrightarrow \quad a / b=c / d .
$$

Note that $a / b$ is reduced if and only if $x \leq b$ and $x \wedge(a \ominus b)=0$ imply $x=0$.

A null quotient $a / a$ is reduced if and only if $a=0$. In an orthoalgebra, a proper quotient $a / b$ is reduced if and only if $b=0$. On the other hand, in a totally ordered effect algebra every proper quotient is reduced.

We say that a finite set $\left\{a_{1} / b_{1}, \ldots, a_{n} / b_{m}\right\}$ of quotients is compatible if and only if $\left\{a_{1}, b_{1}, \ldots, a_{n}, b_{n}\right\}$ is a compatible set.

The aim of this section is to show that for every pairwise orthogonal finite set $\mathbf{f}$ of quotients in a complete lattice-ordered effect algebra there exists a compatible pairwise orthogonal finite set $\mathbf{f}_{R}$ of reduced quotients with $\mathbf{f} \equiv \mathbf{f}_{R}$.

EXAMPLE 11. A quotient $a / b \in Q\left([0,1]^{[0,1]}\right)$ is reduced if and only if

$$
a(x)=b(x) \quad \Longrightarrow \quad a(x)=b(x)=0 .
$$

An effect algebra $E$ is sharply dominating if and only if, for every $x \in E$, the element $x^{\uparrow}$ defined by

$$
x^{\uparrow}=\bigwedge\{t: t \in[x, 1] \cap S(E)\}
$$

exists and is sharp. It is easy to see that in a sharply dominating effect algebra $E$, the element $x^{\downarrow}$ defined by

$$
x^{\downarrow}=\bigvee\{t: t \in[0, x] \cap S(E)\}
$$

exists and is sharp for all $x \in E$. Moreover, we have $x^{\dagger \prime}=x^{\prime \downarrow}$ and $x^{\downarrow^{\prime}}=x^{\prime \uparrow}$. We say that $x^{\uparrow}$ is the sharp cover of $x$ and that $x^{\downarrow}$ is the sharp kernel of $x$. In his paper [3], Cattaneo proved that for every sharply dominating effect algebra the set of all sharp elements forms a subeffect algebra which is an orthoalgebra. See [16] for another version of the proof.

EXAMPLE 12. The lattice-ordered effect algebra $D^{B}$ from Example 4 is sharply dominating, even if $B$ is incomplete. We have

$$
\begin{aligned}
& \left\langle x_{1}, x_{2}\right\rangle^{\uparrow}=\left\langle x_{1} \vee x_{2}, x_{1} \vee x_{2}\right\rangle \quad \text { and } \\
& \left\langle x_{1}, x_{2}\right\rangle^{\downarrow}=\left\langle x_{1} \wedge x_{2}, x_{1} \wedge x_{2}\right\rangle .
\end{aligned}
$$


We say that an effect algebra $E$ is orthocomplete if and only if every chain has a supremum in $E$. See [23] and [22] for results on orthocomplete effect algebras. A lattice-ordered effect algebra is orthocomplete if and only if it is a complete lattice.

PROPOSITION 5.1. ([21, Corollary 5]) Every orthocomplete homogeneous effect algebra is sharply dominating. Moreover, for every block $M, x \in M$ implies that $\left[x^{\downarrow}, x\right],\left[x, x^{\uparrow}\right] \subseteq M$.

Since all complete lattice-ordered effect algebras are orthocomplete and homogeneous, we may apply Proposition 5.1 for them. Note that Proposition 5.1 implies that every subset of a complete lattice-ordered effect algebra of the form $\left[x^{\downarrow}, x\right] \cup\left[x, x^{\uparrow}\right]$ is compatible.

LEMMA 5.2. Let $E$ be a complete lattice-ordered effect algebra. For all $y \in E$, $y^{\prime \downarrow}=y^{\uparrow \prime}$ is the greatest element of the set

$$
\left\{x \in E: x \leq y^{\prime} \text { and } y \wedge x=0\right\} .
$$

PROOF. Let us prove that $y^{\prime \downarrow}$ is the upper bound of the set (5.1). For every $x$, $x \leq y^{\prime}$. Therefore, $x \leftrightarrow y$ and there is a block $M \supseteq\{y, x\}$. By [21, Lemma 1], $y \wedge x=0$ implies that $y^{\uparrow} \wedge x=0$. Since $M$ is an MV-effect algebra, this implies that $x \leq y^{\uparrow \prime}=y^{\prime \downarrow}$.

Since $y^{\prime} \leq y^{\prime}$ and

$$
y \wedge y^{\downarrow}=y \wedge y^{\uparrow \prime} \leq y^{\uparrow} \wedge y^{\uparrow \prime}=0
$$

$y^{\downarrow}$ belongs to (5.1).

PROPOSITION 5.3. Let $E$ be a complete lattice-ordered effect algebra and let $a / b \in Q(E)$. The following are equivalent:

(i) $a / b$ is reduced;

(ii) $b \wedge(a \ominus b)^{\uparrow \prime}=0$;

(iii) $a \leq(a \ominus b)^{\uparrow}$;

(iv) $b \leq(a \ominus b)^{\uparrow} \ominus(a \ominus b)$.

PROOF. (i) $\Longrightarrow$ (ii): Suppose that $x \leq b, x \leq(a \ominus b)^{\uparrow^{\prime}}$. Since $x \leq b, x \leq(a \ominus b)^{\prime}$. By Lemma 5.2, $x \leq(a \ominus b)^{\uparrow \prime}$ implies that $x \wedge(a \ominus b)=0$. Since $a / b$ is reduced, this implies that $x=0$.

(ii) $\Longrightarrow$ (iii): Let $M$ be a block of $E$ with $a, b \in M$. By Proposition 5.1, $(a \ominus b)^{\uparrow} \in M$ and hence $(a \ominus b)^{\uparrow \prime} \in M$. Since $(a \ominus b)^{\uparrow \prime}$ is sharp, $(a \ominus b)^{\uparrow \prime}$ is central in $M$. Thus, we may compute

$a \wedge(a \ominus b)^{\uparrow^{\prime}}=((a \ominus b) \oplus b) \wedge(a \ominus b)^{{ }^{\prime}}=\left((a \ominus b) \wedge(a \ominus b)^{{ }^{\prime}}\right) \oplus\left(b \wedge(a \ominus b)^{{ }^{\prime}}\right)$. 
By Lemma 5.2, $(a \ominus b) \wedge(a \ominus b)^{\uparrow \prime}=0$. By assumption, $b \wedge(a \ominus b)^{\uparrow \prime}=0$. Since $a \wedge(a \ominus b)^{\uparrow \prime}=0$ and $M$ is an MV-effect algebra, $a \leq(a \ominus b)^{\uparrow \prime \prime}=(a \ominus b)^{\uparrow}$.

(iii) $\Longrightarrow$ (iv): We see that $a=(a \ominus b) \oplus b \leq(a \ominus b)^{\uparrow}$, hence $b \leq(a \ominus b)^{\uparrow} \ominus(a \ominus b)$.

(iv) $\Longrightarrow$ (i): Suppose that $x \leq b, x \wedge(a \ominus b)=0$. Since $x \leq b \leq(a \ominus b)^{\uparrow} \ominus(a \ominus b)$, we have $x \leq(a \ominus b)^{\prime}$. By Lemma 5.2, $x \leq(a \ominus b)^{\prime}$ and $x \wedge(a \ominus b)=0$ imply $x \leq(a \ominus b)^{\prime \downarrow}=(a \ominus b)^{\uparrow \prime}$. Since $(a \ominus b)^{\uparrow} \wedge(a \ominus b)^{{ }^{\prime}}=0, x=0$.

The following lemma is crucial.

LEMMA 5.4. Let $E$ be a complete lattice-ordered effect algebra. Let $a / b$ be $a$ reduced quotient of $E$ and let $M$ be a block of $E$ with $a \ominus b \in M$. Then $a, b \in M$.

ProOF. By Proposition 5.3, $a \leq(a \ominus b)^{\uparrow}$. This implies that $a \in\left[a \ominus b,(a \ominus b)^{\uparrow}\right]$. Therefore, by Proposition 5.1, $a \in M$. Since $a,(a \ominus b) \in M, b=a \ominus(a \ominus b) \in M$.

COROLLARY 5.5. Let $E$ be a complete lattice-ordered effect algebra, let $\mathbf{f}$ be a finite set of reduced quotients of $E$ such that $\{a \ominus b: a / b \in \mathbf{f}\}$ is compatible. Then $\mathbf{f}$ is a compatible set of quotients.

Proof. Let $a / b, c / d \in \mathbf{f}$. Since $a \ominus b \leftrightarrow c \ominus d$, there exists a block $M$ with $a \ominus b, c \ominus d \in M$. By Lemma 5.4, $a, b, c, d \in M$. Therefore, $\{a / b, c / d\}$ is a compatible set of quotients. Thus, $\mathbf{f}$ is a compatible set of quotients.

COROLLARY 5.6. Every reduced pairwise orthogonal finite set of quotients of a complete lattice-ordered effect algebra is compatible.

PROOF. This follows from Corollary 5.5.

EXAMPLE 13. If $a / b$ is a quotient of $[0,1]^{[0,1]}$ then $a^{R} / b^{R}$ is given by

$$
a^{R}(x)=\left\{\begin{array}{ll}
a(x) & \text { if } a(x)>b(x), \\
0 & \text { if } a(x)=b(x),
\end{array} \quad b^{R}(x)= \begin{cases}b(x) & \text { if } a(x)>b(x), \\
0 & \text { if } a(x)=b(x)\end{cases}\right.
$$

(See Figure 3.)

Let $E$ be a complete lattice-ordered effect algebra. Let us introduce a mapping $R: Q(E) \rightarrow Q(E)$, given by $a / b \mapsto a^{R} / b^{R}$, where $a^{R}=a \wedge \cdot(a \ominus b)^{\uparrow}$ and $b^{R}=b \wedge(a \ominus b)^{\uparrow}$. We say that $a^{R} / b^{R}$ is the reduct of $a / b$.

PROPOSITION 5.7. Let $E$ be a complete lattice-ordered effect algebra. For every $a / b \in Q(E), a / b \searrow a^{R} / b^{R}$ and $a^{R} / b^{R}$ is reduced. 

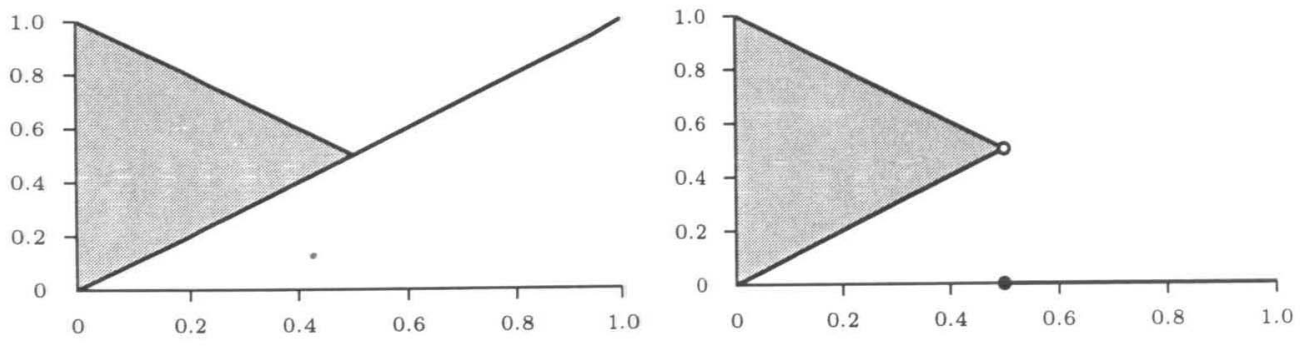

FIGURE 3. $a / b$ and $a^{R} / b^{R}$ in $[0,1]^{[0,1]}$

Proof. Obviously, $a^{R} \leq a$ and $b^{R} \leq b$. Let $M$ be a block with $a, b \in M$. By Proposition 5.1, $(a \ominus b)^{\uparrow} \in M$. Since $(a \ominus b)^{\uparrow}$ is sharp, $(a \ominus b)^{\uparrow}$ is central in $M$. Therefore,

$$
\begin{aligned}
a \ominus a^{R} & =a \ominus\left(a \wedge(a \ominus b)^{\uparrow}\right)=a \wedge(a \ominus b)^{{ }^{\prime}}=(b \oplus(a \ominus b)) \wedge(a \ominus b)^{{ }^{\prime}} \\
& =\left(b \wedge(a \ominus b)^{\uparrow^{\prime}}\right) \oplus\left((a \ominus b) \wedge(a \ominus b)^{\uparrow^{\prime}}\right)=b \wedge(a \ominus b)^{{ }^{\prime}} \\
& =b \ominus\left(b \wedge(a \ominus b)^{\uparrow}\right)=b \ominus b^{R} .
\end{aligned}
$$

Moreover,

$$
(a \ominus b) \wedge\left(a \ominus a^{R}\right)=(a \ominus b) \wedge a \wedge(a \ominus b)^{\uparrow \prime}=0 .
$$

Let us prove that $a^{R} / b^{R}$ is reduced. By Proposition 5.3, this is equivalent to $b^{R} \wedge\left(a^{R} \ominus b^{R}\right)^{\uparrow \prime}=0$. Since $a / b \searrow a^{R} / b^{R}, a \ominus b=a^{R} \ominus b^{R}$. Thus,

$$
b^{R} \wedge\left(a^{R} \ominus b^{R}\right)^{\uparrow \prime}=b \wedge(a \ominus b)^{\uparrow} \wedge(a \ominus b)^{\uparrow \prime}=0 .
$$

PROPOSITION 5.8. Let $E$ be a complete lattice ordered effect algebra and let $a / b, c / d \in Q(E)$. Then $a / b \equiv c / d$ if and only if $a^{R} / b^{R}=c^{R} / d^{R}$.

PROOF. If $a^{R} / b^{R}=c^{R} / d^{R}$ then $a / b \searrow a^{R} / b^{R}=c^{R} / d^{R} \nearrow c / d$.

Suppose that $a / b \nearrow c / d$. By Proposition 4.3,a↔d so there is a block $M$ with $\{a, b, c, d\} \subseteq M$. By Proposition 5.1, $(c \ominus d)^{\uparrow} \in M \cap S(E)=C(M)$. Therefore,

$$
\begin{aligned}
c^{R} & =c \wedge(c \ominus d)^{\uparrow}=((c \ominus a) \oplus a) \wedge(c \ominus d)^{\uparrow} \\
& =\left((c \ominus a) \wedge(c \ominus d)^{\uparrow}\right) \oplus\left(a \wedge(c \ominus d)^{\uparrow}\right) .
\end{aligned}
$$

By Lemma 5.2, $c \ominus a \leq(c \ominus d)^{\prime}$ and $(c \ominus a) \wedge(c \ominus d)=0$ imply that

$$
\text { . } c \ominus a \leq(c \ominus d)^{\downarrow}=(c \ominus d)^{\dagger} \text {. }
$$

Therefore, $(c \ominus a) \wedge(c \ominus d)^{\uparrow}=0$ and

$$
c^{R}=a \wedge(c \ominus d)^{\uparrow}=a \wedge(a \ominus b)^{\uparrow}=a^{R} .
$$

As a consequence, $d^{R}=c^{R} \ominus(c \ominus d)=a^{R} \ominus(a \ominus b)=b^{R}$. 
Let $E$ be a complete lattice-ordered effect algebra. For a finite $n$-element set $\mathbf{f}=\left\{a_{1} / b_{1}, \ldots, a_{n} / b_{n}\right\}$ we write $\mathbf{f}^{R}=\left\{a_{1}^{R} / b_{1}^{R}, \ldots, a_{n}^{R} / b_{n}^{R}\right\}$.

PROPOSITION 5.9. Let $E$ be a complete lattice-ordered effect algebra. Let $\mathbf{f}$ be a finite pairwise orthogonal set of quotients. Then $\mathbf{f} \equiv \mathbf{f}^{R}$ and $\mathbf{f}^{R}$ is a pairwise orthogonal compatible set of quotients.

PROOF. By Lemma $4.6, \mathbf{f} \equiv \mathbf{f}^{R}$. By Lemma $4.5, \mathbf{f}^{R}$ is pairwise orthogonal. By Corollary $5.5, \mathbf{f}^{R}$ is compatible.

\section{Compatible sets of quotients}

In this section we are going to prove a restriction of Theorem 4.7 for compatible events, (see Proposition 6.6.) Using Proposition 5.9, it is then possible to extend the result to the general case.

Let $D$ be a bounded distributive lattice. Up to isomorphism, there exists a unique Boolean algebra $B(D)$ such that $D$ is a 0,1 -sublattice of $B(D)$ and $D$ generates $B(D)$ as a Boolean algebra. This Boolean algebra is called an R-generated Boolean algebra. We refer to [13, Section II.4] for an overview of results concerning R-generated Boolean algebras. See also [17] and [27]. For every element $x$ of $B(D)$, there exists a finite chain $x_{1} \leq \cdots \leq x_{n}$ in $D$ such that $x=x_{1}+\cdots+x_{n}$, where + denotes the symmetric difference, as in Boolean rings. We then say that $\left\{x_{i}\right\}_{i=1}^{n}$ is a $D$-chain representation of $x$. It is easy to see that every element of $B(D)$ has a $D$-chain representation of even length.

Note that if $D_{1}$ is a 0,1 -sublattice of a distributive lattice $D_{2}$ then $B\left(D_{1}\right)$ is a subalgebra of $B\left(D_{2}\right)$.

LemMA 6.1. ([20, Lemma 7]). Let $L$ be a finite 0, 1-sublattice of an $M V$-effect algebra $M$. The mapping $\phi_{L}: B(L) \rightarrow M$ given by

$$
\phi_{L}(x)=\bigoplus_{i=1}^{n}\left(x_{2 i} \ominus x_{2 i-1}\right),
$$

where $\left\{x_{i}\right\}_{i=1}^{2 n}$ is a L-chain representation of $x$, is a faithful surjective homomorphism of effect algebras. The value of $\phi_{L}(x)$, as given by (6.1), does not depend on the choice of $\left\{x_{i}\right\}_{i=1}^{2 n}$.

Note that, since every compatible 0,1 -sublattice $L$ of a lattice-ordered effect algebra is a sublattice of some block $M$, Lemma 6.1 is true even if we merely suppose that $L$ is a compatible 0, 1-sublattice of a lattice-ordered effect algebra. 
Let $L$ be a lattice. An element $a$ of $L$ is join-irreducible if and only if $a=b \vee c$ implies that $a=b$ or $a=c$; it is meet-irreducible if and only if $a=b \wedge c$ implies that $a=b$ or $a=c$. The set of all nonzero join-irreducible elements of a lattice $L$ is denoted by $J(L)$ and the set of all non-unit meet-irreducible elements of a lattice $L$ is denoted by $M(L)$.

Let $L$ be a finite distributive lattice. Then the mapping $r: L \rightarrow 2^{J(L)}$ given by $r(x)=\{a \in J(L): a \leq x\}$ is a 0,1 -embedding of $L$ into $2^{J(L)}$. Since, for every finite distributive lattice $L, r(L)$ R-generates $2^{J(L)}$, the injective mapping $r: L \rightarrow 2^{J(L)}$ uniquely extends to an isomorphism of Boolean algebras $\hat{r}: B(L) \rightarrow 2^{J(L)}$.

In what follows, $\succ_{P}$ denotes the usual covering relation on a poset $P$, so that $a \succ_{P} b$ if and only if $b$ is a maximal element of the set $\{x \in P: x<a\}$. In a finite distributive lattice $L$, we have $a \succ_{L} b$ if and only if $\hat{r}(a) \backslash \hat{r}(b)$ is a singleton.

Let $L$ be a finite distributive lattice. We have $a \in J(L)$ if and only if there is a unique $b$ such that $a \succ_{L} b$. Therefore $\left\{a+b: a \succ_{L} b, a \in J(L)\right\}$ is the set of all atoms of $B(L)$.

Let $L$ be a finite 0,1 -sublattice of a lattice-ordered effect algebra $E$, and let $\mathbf{f}=\left\{a_{1} / b_{1}, \ldots, a_{n} / b_{m}\right\}$ be a compatible set of quotients such that $\mathbf{f} \subseteq Q(L)$. We write

$$
+\mathbf{f}=a_{1}+b_{1}+\cdots+a_{n}+b_{n}
$$

where the + on the right-hand side is taken in $B(L)$.

LEMMA 6.2. Let $E$ be a complete lattice-ordered effect algebra and let $\{a / b, c / d\}$ be compatible. Let $L \supseteq\{a, b, c, d\}$ be a finite compatible 0,1 -sublattice of $E$. Then $a / b \equiv c / d$ implies that $a+b=c+d$ in $B(L)$.

Proof. By Proposition 5.8, $a / b \equiv c / d$ implies that $a^{R} / b^{R}=c^{R} / d^{R}$. Let $M$ be a block of $E$ such that $L \subseteq M$. Since $a \ominus b \in M$, Lemma 5.4 implies $a^{R} / b^{R} \in Q(M)$. Therefore $\left\{a / b, c / d, a^{R} / b^{R}=c^{R} / d^{R}\right\}$ is a compatible set of quotients. Let $L_{1}$ be a finite compatible lattice such that $L \subseteq L_{1} \subseteq M$ and $\left\{a^{R}, b^{R}\right\} \subseteq L_{1}$.

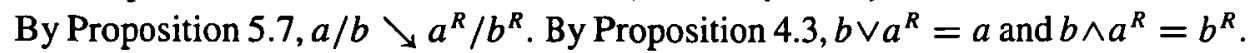
Therefore, we may calculate in $B\left(L_{1}\right)$ that

$$
a+b=\left(b \vee a^{R}\right)+b=a^{R}+\left(b \wedge a^{R}\right)=a^{R}+b^{R} .
$$

Similarly, c./d $\searrow c^{R} / d^{R}$ implies that $c+d=c^{R}+d^{R}$. Therefore

$$
a+b=a^{R}+b^{R}=c^{R}+d^{R}=c+d
$$

in $B\left(L_{1}\right)$ and, since $B(L)$ is a subalgebra of $B\left(L_{1}\right), a+b=c+d$ in $B(L)$. 
PROPOSITION 6.3. Let $E$ be a complete lattice-ordered effect algebra and let $\{a / b, c / d\}$ be compatible. Let $L \supseteq\{a, b, c, d\}$ be a finite compatible 0,1 -sublattice of $E$. Suppose that $a \succ_{L} b$. Then $a / b \equiv c / d$ if and only if $a+b=c+d$ in $B(L)$.

PROOF. Suppose that $a+b=c+d$. Since $a \succ_{L} b, \hat{r}(a) \backslash \hat{r}(b)$ is a singleton. Let $e \in \hat{r}(a) \backslash \hat{r}(b)$. Since $e$ is join-irreducible and nonzero, there is a single element $f \in L$ such that $e \succ_{L} f$. We claim that $a / b \searrow e / f$. Indeed,

$$
a \wedge(b \vee e)=(a \wedge b) \vee(a \wedge e)=b \vee e,
$$

hence $a \geq b \vee e \geq b$. Since $a \succ_{L} b$, we have either $b \vee e=a$ or $b \vee e=b$. However, $b \vee e=b$ implies that $e \in \hat{r}(b)$, which contradicts $e \in \hat{r}(a) \backslash \hat{r}(b)$. Therefore $b \vee e=a$.

Since $L$ is distributive, the intervals $[b, b \vee e]$ and $[b \wedge e, e]$ are isomorphic. As $b \vee e=a \succ_{L} b, e \succ_{L} b \wedge e$. Since $e$ is join-irreducible and nonzero, $e$ covers exactly one element, hence $b \wedge e=f$. We have proved that $b \vee e=a$ and $a \wedge e=f$. By Proposition 4.3, $a / b \searrow e / f$.

Since $a+b=c+d, \hat{r}(a) \backslash \hat{r}(b)=\hat{r}(c) \backslash \hat{r}(d)$ and $\hat{r}(c) \backslash \hat{r}(d)$ is a singleton. This implies that $c \succ_{L} d$ and, as for $a / b$, we deduce $c / d \searrow e / f$. Therefore, $a / b \searrow e / f \nearrow c / d$ and $a / b \equiv c / d$.

The converse implication follows by Lemma 6.2.

LEMMA 6.4. Let $E$ be a complete lattice-ordered effect algebra and let $\{a / b, c / d\}$ be compatible. Let $L \supseteq\{a, b, c, d\}$ be a finite compatible 0,1 -sublattice of $E$. Then $a / b$ and $c / d$ are orthogonal if and only if $a+b$ and $c+d$ are disjoint in $B(L)$.

Proof. Suppose that $a / b$ and $c / d$ are orthogonal and that $a+b$ and $c+d$ are not disjoint in $B(L)$. Then there exists $e \in(\hat{r}(a) \backslash \hat{r}(b)) \cap(\hat{r}(c) \backslash \hat{r}(d))$.

Let $a_{0} / b_{0}, \ldots, a_{n} / b_{n}$ and $c_{0} / d_{0}, \ldots, c_{k} / d_{k}$ be sequences of quotients of $L$ such that

$$
a=a_{0} \succ_{L} b_{0}=a_{1} \succ_{L} b_{1}=a_{2} \succ_{L} \cdots \succ_{L} b_{n-1}=a_{n} \succ_{L} b_{n}=b
$$

and

$$
c=c_{0} \succ_{L} d_{0}=c_{1} \succ_{L} d_{1}=c_{2} \succ_{L} \cdots \succ_{L} d_{k-1}=c_{k} \succ_{L} d_{k}=d .
$$

In $2^{J(L)}$, we have

$$
\hat{r}(a) \backslash \hat{r}(b)=\dot{\cup}_{i=0}^{n}\left(\hat{r}\left(a_{i}\right) \backslash \hat{r}\left(b_{i}\right)\right)
$$

and similarly for $\hat{r}(c) \backslash \hat{r}(d)$. Therefore $e \in\left(\hat{r}\left(a_{i}\right) \backslash \hat{r}\left(b_{i}\right)\right) \cap\left(\hat{r}\left(c_{j}\right) \backslash \hat{r}\left(d_{j}\right)\right)$ for some $i, j$. Since $a_{i} \succ_{L} b_{i}$ and $c_{j} \succ_{L} d_{j}$, this implies that $\hat{r}\left(a_{i}\right) \backslash \hat{r}\left(b_{i}\right)=\hat{r}\left(c_{j}\right) \backslash \hat{r}\left(d_{j}\right)$, which means $a_{i}+b_{i}=c_{j}+d_{j}$. By Proposition 6.3, $a_{i} / b_{i} \equiv c_{j} / d_{j}$. This contradicts $a / b \perp c / d$. 
Suppose that $a+b$ and $c+d$ are disjoint in $B(L)$. Let $x / y, z / w \in Q(E)$ be such that

$$
a / b \sqsupseteq x / y \equiv z / w \sqsubseteq c / d
$$

Obviously $\{a, b, x, y\}$ and $\{c, d, z, w\}$ are compatible sets. Since $x \ominus y=z \ominus w \leq a, c$ and $x \ominus y=z \ominus w \leq b^{\prime}, d^{\prime}$, it follows that $\{a, b, c, d, x \ominus y=z \ominus w\}$ is compatible as well. By Lemma 5.4, this implies that the sets of quotients

$$
\begin{gathered}
\mathbf{f}_{1}:=\left\{a / b, x / y, a^{R} / b^{R}, x^{R} / y^{R}\right\}, \quad \mathbf{f}_{2}:=\left\{c / d, z / w, c^{R} / d^{R}, z^{R} / w^{R}\right\}, \quad \text { and } \\
\mathbf{g}:=\left\{a / b, c / d, a^{R} / b^{R}, c^{R} / d^{R}, x^{R} / y^{R}\right\}
\end{gathered}
$$

are compatible. Moreover, by Proposition 5.8, $x^{R} / y^{R}=z^{R} / w^{R}$. Let $L_{1}, L_{2}$ and $K$ be finite compatible 0,1 -sublattices of $E$ such that $\mathbf{f}_{1} \subseteq Q\left(L_{1}\right), \mathbf{f}_{2} \subseteq Q\left(L_{2}\right)$ and $\mathrm{g} \subseteq Q(K)$.

Obviously, $a+b \geq_{B\left(L_{1}\right)} x+y$. By Lemma $6.2, a+b=_{B\left(L_{1}\right)} a^{R}+b^{R}$ and $x+y=_{B\left(L_{1}\right)} x^{R}+y^{R}$. Therefore $a^{R}+b^{R} \geq_{B\left(L_{1}\right)} x^{R}+y^{R}$. Since $B\left(L_{1} \cap K\right)$ is a subalgebra of $B\left(L_{1}\right)$, we have $a^{R}+b^{R} \geq_{B\left(L_{1} \cap K\right)} x^{R}+y^{R}$. Since $B\left(L_{1} \cap K\right)$ is a subalgebra of $B(K)$, this implies that $a^{R}+b^{R} \geq_{B(K)} x^{R}+y^{R}$. Similarly, $c^{R}+d^{R} \geq_{B(K)} z^{R}+w^{R}=x^{R}+y^{R}$. Since $a+b$ and $c+d$ are disjoint in $B(L)$, they are disjoint in $B(L \cap K)$ and hence also in $B(K)$. By Lemma 6.2, $a^{R}+b^{R}={ }_{B(K)} a+b$ and $c^{R}+d^{R}={ }_{B(K)} c+d$. Thus $a^{R}+b^{R}$ and $c^{R}+d^{R}$ are disjoint elements of $B(K)$. This implies that $x^{R}+y^{R}=0$, so $x^{R}=y^{R}$ and hence $x=y$.

PROPOSITION 6.5. Let $E$ be a complete lattice-ordered effect algebra. Let $\mathbf{f}=\left\{a_{1} / b_{1}, \ldots, a_{n} / b_{n}\right\} \subseteq Q(E)$ be a compatible set of quotients. Let $L$ be a finite compatible 0,1 -sublattice of $E$ with $\left\{a_{1}, b_{1}, \ldots, a_{n}, b_{n}\right\} \in L$. Then $\mathbf{f}$ is orthogonal if and only if, for all $i \neq j, a_{i}+b_{i}$ and $a_{j}+b_{j}$ are disjoint in $B(L)$.

PROOF. $(\Leftrightarrow)$ : This follows from Lemma 6.4 .

$(\Leftarrow)$ : By Lemma 6.4 , the elements of $\mathbf{f}$ are pairwise disjoint. It remains to prove that

$$
|\mathbf{f}|=\left(a_{1} \ominus b_{1}\right) \oplus \cdots \oplus\left(a_{n} \ominus b_{n}\right)
$$

exists. By assumption, the sum

$$
\left(a_{1}+b_{1}\right) \oplus \cdots \oplus\left(a_{n}+b_{n}\right)
$$

exists in the effect algebra $B(L)$. Since $\phi_{L}$ is a morphism of effect algebras, the sum

$$
\phi_{L}\left(a_{1}+b_{1}\right) \oplus \cdots \oplus \phi_{L}\left(a_{n}+b_{n}\right)
$$

exists in $E$. It remains to observe that, for all $i, \phi_{L}\left(a_{i}+b_{i}\right)=a_{i} \ominus b_{i}$. 
It is now clear that, for every finite compatible 0,1 -sublattice $L$ of a complete lattice ordered effect algebra $E$,

$$
\left\{a / b: a \succ_{L} b, a \in J(L)\right\}
$$

is a compatible test of $\Omega(E)$. On the other hand, for a finite compatible and orthogonal set of quotients $\mathbf{f}=\left\{a_{1} / b_{1}, \ldots, a_{n} / b_{n}\right\}$ we have

$$
+\mathbf{f}=\left(a_{1}+b_{1}\right) \dot{V} \cdots \dot{\vee}\left(a_{n}+b_{n}\right)
$$

in every $B(L)$ with $\mathbf{f} \subseteq Q(L)$, where $L$ is a finite compatible 0 , 1-sublattice of $E$.

PROPOSITION 6.6. Let $E$ be a complete lattice-ordered effect algebra. Let $\mathbf{f}$ be a finite compatible set of quotients of $E$. Then the following are equivalent:

(a) $\mathbf{f}$ is an event of $\Omega(E)$;

(b) $\mathbf{f}$ is an orthogonal set of quotients;

(c) $\mathbf{f}$ is pairwise orthogonal.

ProOF. (a) implies (b) and (b) implies (c) by definition.

To show that (c) implies (a), we shall prove that there exists a compatible and orthogonal finite set $\mathbf{t} \supseteq \mathbf{f}$ with $|\mathbf{t}|=1$. Let $\mathbf{f}=\left\{a_{1} / b_{1}, \ldots, a_{n} / b_{n}\right\}$. Let $L$ be a finite compatible 0,1 -sublattice of $E$ with $\left\{a_{1}, b_{1}, \ldots, a_{n}, b_{n}\right\} \subseteq L$. Let $\left(c_{i}\right)_{i=1}^{2 k}$ be an $L$-chain representation of the complement of $a_{1}+b_{1}+\cdots+a_{n}+b_{n}$ in $B(L)$. By Proposition 6.5,

$$
\mathbf{t}=\left\{a_{1} / b_{1}, \ldots, a_{n} / b_{n}, c_{2} / c_{1}, \ldots, c_{2 k} / c_{2 k-1}\right\}
$$

is orthogonal. By Lemma 6.1, we have $|\mathbf{t}|=1$.

COROLLARY 6.7. Let $E$ be a complete lattice-ordered effect algebra. Let $\mathbf{f} \subseteq Q(E)$ and $\mathbf{g} \subseteq Q(E)$ be events of $\Omega(E)$ such that $\mathbf{f} \cup \mathbf{g}$ is compatible. Let $L$ be $a$ finite compatible 0, 1-sublattice of $E$ with $\mathbf{f}, \mathbf{g} \subseteq Q(L)$. Then $\mathbf{f} \perp \mathbf{g}$ if and only if $+\mathbf{f} \perp_{B(L)}+\mathbf{g}$ and $\mathbf{f}$ loc $\mathbf{g}$ if and only if $+\mathbf{f} \perp_{B(L)}+\mathbf{g}$ and $\phi_{L}((+\mathbf{f}) \dot{v}(+\mathbf{g}))=1$.

PROOF. This follows from Propositions 6.5 and 6.6.

PROOF (Proof of Theorem 4.7). Let $f$ be a finite pairwise orthogonal set of quotients. Since $\mathbf{f} \equiv \mathbf{f}^{R}, \mathbf{f}^{R}$ is pairwise orthogonal. By Corollary $5.6, \mathbf{f}^{R}$ is compatible. By Proposition $6.6, \mathbf{f}^{R}$ is an event of $\Omega(E)$, therefore there exists a test $t_{0} \supseteq \mathbf{f}^{R}$. Put $\mathbf{t}=\mathbf{f} \cup\left(\mathbf{t}_{0} \backslash \mathbf{f}^{R}\right)$. By Lemma $4.6, \mathbf{t} \equiv \mathbf{t}_{0}$. By Lemma $4.5, \mathbf{t}$ is a test. 
PROPOSITION 6.8. Let $E$ be a complete lattice-ordered effect algebra and let $\mathbf{f}, \mathbf{g}$ be events of $\Omega(E)$. Then $\mathbf{f} \perp \mathbf{g}$ if and only if, for all $x / y \in \mathbf{f}$ and $z / w \in \mathbf{g}$, we have $x / y \perp z / w$.

PRoOF. $\mathbf{f} \perp \mathbf{g}$ if and only if $\mathbf{f} \cap \mathbf{g} \in \mathscr{N}$ and $\mathbf{f} \cup \mathbf{g}$ is an event of $\Omega(E)$. The rest follows by Theorem 4.7 .

\section{7. $\Omega(E)$ is algebraic}

Let $\mathbf{f}$ be a finite orthogonal set of quotients and let $z / w \in Q(E)$. We say that $z / w$ is covered by $\mathbf{f}$ if and only if there are $z_{1} / w_{1}, \ldots, z_{n} / w_{n}$ such that

- $z=z_{1}$,

- $w_{n}=w$,

- for all $1 \leq i<n, w_{i}=z_{i+1}$,

- for all $1 \leq i \leq n$, there are $c / d$ and $e / f$ such that $z_{i} / w_{i} \equiv c / d \sqsubseteq e / f \in \mathbf{f}$.

PROPOSITION 7.1. Let $E$ be a complete lattice-ordered effect algebra and let $\mathbf{t}$ be $a$ test of $\Omega(E)$. Let $z / w$ be such that, for all $e / f \in \mathbf{t}, z \ominus w \leftrightarrow e \ominus f$. Then $z / w$ is covered by $\mathbf{t}$.

PROOF. Let us write $\mathbf{t}=\left\{e_{1} / f_{1}, \ldots, e_{m} / f_{m}\right\}$. By Corollary $5.5, \mathbf{t}^{R} \cup\left\{z^{R} / w^{R}\right\}$ is a compatible set of quotients. Let $L$ be a finite compatible 0,1 -sublattice of $E$ with $\mathbf{t}^{R} \cup\left\{z^{R} / w^{R}\right\} \subseteq Q(L)$. By Lemma 6.1,

$$
\phi_{L}\left(\left(e_{1}^{R}+f_{1}^{R}\right) \dot{\vee} \cdots \dot{\vee}\left(e_{m}^{R}+f_{m}^{R}\right)\right)=\left(e_{1}^{R} \ominus f_{1}^{R}\right) \oplus \cdots \oplus\left(e_{m}^{R} \ominus f_{m}^{R}\right)=|\mathbf{t}|=1 .
$$

Since $\phi_{L}$ is faithful, this implies that $\left(e_{1}^{R}+f_{1}^{R}\right) \dot{v} \cdots \dot{v}\left(e_{m}^{R}+f_{m}^{R}\right)=1$.

Let $z_{1} / w_{1}, \ldots, z_{n} / w_{n} \subseteq Q(L)$ be such that

- $z^{R}=z_{1}$,

- $w_{n}=w^{R}$,

- for all $1 \leq i<n, z_{i}=w_{i+1}$,

- for all $1 \leq i \leq n, z_{i}>_{L} w_{i}$.

In $B(L)$, we have

$$
z^{R}+w^{R}=\left(z_{1}+w_{1}\right) \dot{v} \cdots \dot{v}\left(z_{n}+w_{n}\right) .
$$

Since each $z_{i}+w_{i}$ is an atom of $B(L)$, we see that for every $1 \leq i \leq n$ there exists some $1 \leq j \leq m$ such that $z_{i}+w_{i} \leq e_{j}^{R}+f_{j}^{R}$. Therefore there exist $c, d \in L$ such that $z_{i}+w_{i}=c+d$ and $e_{j}^{R} \geq c \succ d \geq f_{j}^{R}$. By Proposition 6.3, $z_{i} / w_{i} \equiv c / d$.

Since $z^{R} / w^{R}$ is covered by $t^{R}, z / w$ is covered by $t$. 
THEOREM 7.2. For every complete lattice-ordered effect algebra $E, \Omega(E)$ is an algebraic generalized test space.

PROOF. Let $\mathbf{f}, \mathbf{g}, \mathbf{h}$ be such that $\mathbf{f} \sim \mathbf{g}, \mathbf{g} \operatorname{loc} \mathbf{h}$. We shall prove that $\mathbf{f} \operatorname{loc} \mathbf{h}$.

There is an event $\mathbf{u}$ such that $\mathbf{f} \operatorname{loc} \mathbf{u}$ and $\mathbf{g} \operatorname{loc} \mathbf{u}$. Since $|\mathbf{f}| \oplus|\mathbf{h}|=1$, it suffices to prove that every pair of quotients $a / b \in \mathbf{f}$ and $c / d \in \mathbf{h}$ is disjoint. Assume the contrary and let $x / y, z / w$ be proper quotients such that

$$
a / b \sqsupseteq x / y \equiv z / w \sqsubseteq c / d .
$$

Since $(x \ominus y) \oplus|\mathbf{u}|$ and $(z \ominus w) \oplus|\mathbf{g}|$ exist and $x \ominus u=z \ominus w$, we see that, for all $e / f \in \mathbf{u} \cup \mathbf{g}, x \ominus y=z \Theta w \leq(e \ominus f)^{\prime}$. Therefore $x \ominus y=z \ominus w \leftrightarrow e \ominus f$. By Proposition 7.1, this implies that $x / y$ is covered by the test $\mathbf{u} \cup \mathbf{g}$. However, since $x / y \sqsubseteq a / b \in \mathbf{f} \perp \mathbf{u}, x / y$ is disjoint with every element of $\mathbf{u}$. Therefore $x / y$ is covered by $g$. In particular, there exists a proper quotient $x_{1} / y_{1} \sqsubseteq x / y$ such that $x_{1} / y_{1} \sqsubseteq p / q$ for some $p / q \in \mathrm{g}$. As $x_{1} / y_{1} \sqsubseteq x / y$ and $x / y \equiv z / w$, there exists a proper quotient $z_{1} / w_{1} \sqsubseteq z / w$ such that $z_{1} / w_{1} \equiv x_{1} / y_{1}$. Obviously $z_{1} \ominus w_{1} \leq z \ominus w$ implies that, for all $e / f \in \mathbf{u} \cup \mathbf{g}, x_{1} \ominus y_{1}=z_{1} \ominus w_{1} \leq(e \ominus f)^{\prime}$ and hence $x_{1} \ominus y_{1}=z_{1} \ominus w_{1} \leftrightarrow e \ominus f$. By Proposition 7.1, this implies that $z_{1} / w_{1}$ is covered by the test $\mathbf{u} \cup \mathbf{g}$. Since $z_{1} / w_{1} \sqsubseteq z / w \sqsubseteq c / d \in \mathbf{h} \perp \mathbf{g}, z_{1} / w_{\mathbf{1}}$ is covered by $\mathbf{u}$. In particular, there is $r / s \in \mathbf{u}$ such that there is a proper quotient $z_{2} / w_{2} \sqsubseteq z_{1} / w_{1}, r / s$. As $z_{1} / w_{1} \equiv x_{1} / y_{1}$, there is a proper quotient $x_{2} / y_{2} \sqsubseteq x_{1} / y_{1}$ such that $x_{2} / y_{2} \equiv z_{2} / w_{2}$. We see that

$$
p / q \sqsupseteq x_{1} / y_{1} \sqsupseteq x_{2} / y_{2} \equiv z_{2} / w_{2} \sqsubseteq z_{1} / w_{1} \sqsubseteq r / s
$$

and $x_{2} / y_{2}$ is proper. This is a contradiction with $r / s \perp p / q$.

For a complete lattice-ordered effect algebra, we denote the orthoalgebra of $\Omega(E)$ by $O(E)$.

COROLLARY 7.3. For every complete lattice-ordered effect algebra $E$, the mapping $\phi_{E}: O(E) \rightarrow E$ given by $\phi_{E}\left([\mathbf{f}]_{\sim}\right)=|\mathbf{f}|$ is a surjective full morphism of effect algebras.

PROOF. It is easy to check that $\phi_{E}$ is a morphism of effect algebras. Let $s, t \in E$ and suppose that $s \oplus t$ exists. Then, in $\Omega(E),\{s / 0\} \perp\{s \oplus t / s\}$ and hence, in $O(E)$, the $\operatorname{sum}[\{s / 0\}]_{\sim} \oplus[\{s \oplus t / s\}]_{\sim}$ exists. Since $\phi_{E}\left([\{s / 0\}]_{\sim}\right)=s$ for all $s \in E, \phi_{E}$ is surjective.

To abbreviate our notations, let us write

- $\mathbf{f} \lesssim \mathbf{g}$ instead of $[\mathbf{f}]_{\sim} \leq[\mathbf{g}]_{\sim}$,

- $a / b \perp \mathbf{f}$ instead of $\{a / b\} \perp \mathbf{f}$, 
- $a / b \lesssim \mathbf{f}$ instead of $\{a / b\} \lesssim \mathbf{f}$.

PROPOSITION 7.4. Let $E$ be a complete lattice-ordered effect algebra and let $\mathbf{f}, \mathbf{g}$ be events of $\Omega(E)$. Then $\mathbf{f} \lesssim \mathbf{g}$ if and only if, for all $a / b \in Q(E), a / b \perp \mathbf{g}$ implies $a / b \perp \mathbf{f}$.

PROOF. Suppose that $\mathbf{f} \lesssim \mathbf{g}$. There is an event $\mathbf{v}$ such that $\mathbf{v} \perp \mathbf{f}$ and $\mathbf{v} \cup \mathbf{f} \sim \mathbf{g}$. If $a / b \perp \mathbf{g}$ then $a / b \perp \mathbf{v} \cup \mathbf{f}$ and $a / b \perp \mathbf{f}$.

Suppose that, for all $a / b \in Q(E), a / b \perp \mathbf{g}$ implies that $a / b \perp \mathbf{f}$. Let $\mathbf{u}$ be a local complement of $\mathbf{g}$. By assumption, every quotient in $\mathbf{u}$ is orthogonal to $\mathbf{f}$. By Proposition 6.8, this implies that $\mathbf{f} \cup \mathbf{u}$ is an event. Let $\mathbf{v}$ be a local complement of $\mathbf{f} \cup \mathbf{u}$. Then $\mathbf{u}$ is a local complement of $\mathbf{f} \cup \mathbf{v}$. Consequently, $\mathbf{f} \cup \mathbf{v} \sim \mathbf{g}$ and $\mathbf{f} \lesssim \mathbf{g}$.

COROLLARY 7.5. Let $E$ be a complete lattice-ordered effect algebra and let $\mathbf{f}, \mathbf{g}$ be events of $\Omega(E)$. Then $\mathbf{f} \sim \mathbf{g}$ if and only if, for all $a / b \in Q(E), a / b \perp \mathbf{f}$ if and only if $a / b \perp \mathbf{g}$.

PROOF. This follows from Proposition 7.4.

PROPOSITION 7.6. Let $E$ be a complete lattice-ordered effect algebra and let $\mathbf{f}, \mathbf{g}$ be events of $\Omega(E)$. Then $\mathbf{f} \lesssim \mathbf{g}$ if and only if, for all $x / y \in \mathbf{f}, x / y \lesssim \mathbf{g}$.

PROOF. Suppose that for all $x / y \in \mathrm{f}$ we have $x / y \lesssim \mathbf{g}$. Let h be a local complement of $\mathbf{g}$. Then $\mathbf{f} \lesssim \mathbf{g}$ if and only if $\mathbf{f} \perp \mathbf{h}$. Let $x / y \in \mathbf{f}$ and $z / w \in \mathbf{h}$. Since $x / y \lesssim \mathbf{g} \perp \mathbf{h} \gtrsim z / w$, we see that $x / y \perp z / w$. By Proposition 6.8, $\mathbf{f} \perp \mathbf{h}$.

\section{Perspectivity of sharp and compatible events}

We say that an event $\mathbf{f}$ of $\Omega(E)$ sharp if and only if $|\mathbf{f}|$ is sharp.

PROPOSITION 8.1. Let $E$ be a complete lattice-ordered effect algebra. Let $\mathbf{g}$ be a sharp event of $\Omega(E)$ and let $\mathbf{f}$ be an event of $\Omega(E)$. Then $\mathbf{f} \lesssim \mathbf{g}$ if and only if $|\mathbf{f}| \leq|\mathbf{g}|$.

ProOF. Obviously, $\mathbf{f} \lesssim \mathbf{g}$ implies that $|\mathbf{f}| \leq|\mathbf{g}|$.

Suppose that $|\mathbf{f}| \leq|\mathbf{g}|$ and that $a / b \perp \mathbf{g}$. By Proposition 7.4, it suffices to prove that $a / b \perp \mathbf{f}$. Suppose that $a / b \not \subset \mathbf{f}$. By Proposition 6.8, this implies that $a / b \not \perp c / d$ for some $c / d \in \mathbf{f}$. As $(a \ominus b) \oplus|\mathbf{g}|$ exists, $(a \ominus b) \oplus(c \ominus d)$ exists. Therefore $a / b$ is not disjoint with $c / d$. In particular, $(a \ominus b) \wedge(c \ominus d)>0$. However, we then have

$$
\begin{aligned}
& 0<(a \ominus b) \wedge(c \ominus d) \leq a \ominus b \leq|\mathbf{g}|^{\prime} \text { and } \\
& 0<(a \ominus b) \wedge(c \ominus d) \leq c \ominus d \leq|\mathbf{f}| \leq|\mathbf{g}| .
\end{aligned}
$$

This is a contradiction with $|\mathrm{g}| \in S(E)$. 
COROLLARY 8.2. Let $E$ be a complete orthomodular lattice. Then the mapping $\psi: O(E) \rightarrow$ E given by $\psi\left([\mathbf{f}]_{\sim}\right)=|\mathbf{f}|$ is an isomorphism.

PROOF. The proof is a trivial application of Proposition 8.1 and is omitted.

PROPOSITION 8.3. Let $E$ be a complete lattice-ordered effect algebra. Let $\mathbf{f}, \mathbf{g}$ be compatible events of $\Omega(E)$ such that $\mathbf{f} \cup \mathbf{g}$ is compatible. Let $L$ be a finite compatible 0,1 -sublattice of $E$ with $\mathbf{f} \cup \mathbf{g} \subseteq Q(L)$. Then $\mathbf{f} \lesssim \mathbf{g}$ if and only if $+\mathbf{f} \leq+\mathbf{g}$ in $B(L)$.

PROOF. Let $\left(c_{i}\right)_{i=1}^{2 k}$ be an $L$-chain representation of the complement of $+\mathrm{g}$ in $B(L)$ and write $\mathbf{h}=\left\{c_{2} / c_{1}, \ldots, c_{2 k} / c_{2 k-1}\right\}$. By Corollary $6.7, \mathbf{g}$ loc $\mathbf{h}$.

Since $\Omega(E)$ is algebraic, $\mathbf{f} \lesssim \mathbf{g}$ is equivalent to $\mathbf{f} \perp \mathbf{h}$. By Corollary $6.7, \mathbf{f} \perp \mathbf{h}$ if and only if $+\mathbf{f} \perp+\mathbf{h}$. Obviously $+\mathbf{f} \perp+\mathbf{h}$ if and only if $+\mathbf{f} \leq+\mathbf{g}$.

COROLlARY 8.4. Let $M$ be a complete $M V$-effect algebra. Then the mapping $\psi: O(M) \rightarrow B(M)$ given by $\psi\left([\mathbf{f}]_{\sim}\right)=+\mathbf{f}$ is an isomorphism of effect algebras.

PROOF. Let us prove that $\psi$ is well-defined: suppose that $\mathbf{f} \sim \mathbf{g}$. Since $M$ is an MV-effect algebra, $\mathbf{f} \cup \mathbf{g}$ is compatible. By Proposition $8.3,+\mathbf{f}=+\mathbf{g}$. Obviously $\psi$ is surjective. Suppose that $\psi(\mathbf{f})=\psi(\mathbf{g})$, which means $+\mathbf{f}=+\mathbf{g}$. By Proposition 8.3, $\mathbf{f} \sim$ g.

It remains to prove that $\phi$ is a homomorphism. Suppose that $[\mathrm{f}]_{\sim} \perp[\mathrm{g}]_{\sim}$. This implies that $\mathbf{f} \perp \mathbf{g}$. Let $L$ be a finite 0,1 -sublattice of $E$ with $\mathbf{f} \cup \mathbf{g} \subseteq Q(L)$. By Corollary 6.7, $\psi(\mathbf{f}) \perp \psi(\mathrm{g})$ and, obviously,

$\psi\left([\mathbf{f}]_{\sim} \oplus[\mathbf{g}]_{\sim}\right)=\psi\left([\mathbf{f} \cup \mathbf{g}]_{\sim}\right)=(+\mathbf{f}) \oplus(+\mathbf{g})=\psi\left([\mathbf{f}]_{\sim}\right) \oplus \psi\left([\mathbf{g}]_{\sim}\right)$.

\section{9. $O(E)$ is a lattice}

Let $\mathbf{f}=\left\{a_{1} / b_{2}, \ldots, a_{n} / b_{n}\right\}$ be a compatible event of $E$. In what follows, $\langle\mathbf{f}\rangle$ denotes the (finite distributive) 0,1 -sublattice of $E$ generated by the set $\left\{a_{1}, b_{1}, \ldots, a_{n}, b_{n}\right\}$.

Let $\mathbf{f}$ be an event of $E$. We denote the test

$$
\left\{a / b: a \in J\left(\left\langle\mathbf{f}^{R}\right\rangle\right), a \succ_{L} b\right\}
$$

by $\mathbf{t}_{\mathbf{r}}$. We write

$$
\mathbf{f}^{*}=\left\{a / b \in \mathbf{t}_{\mathbf{f}}: a / b \perp \mathbf{f}\right\} .
$$

We have $\mathbf{f} \sim \mathbf{f}^{R}$ and $\mathbf{f}^{R}$ loc $\mathbf{f}^{*}$. Since $\Omega(E)$ is algebraic, $\mathbf{f} \operatorname{loc} \mathbf{f}^{*}$.

PROPOSITION 9.1. Let $E$ be a complete lattice-ordered effect algebra. Let $\mathbf{f}$ be an event of $\Omega(E)$ and let $a / b \in Q(E)$. Then $a / b \perp \mathbf{f}$ if and only if $a / b$ is covered by $\mathbf{f}^{*}$. 
PROOF. Suppose that $a / b \perp \mathbf{f}$. We shall prove that $a / b$ is covered by $\mathbf{f}^{*}$. For all $c / d \in \mathbf{f}, a \ominus b \leftrightarrow c \ominus d$. Therefore, by Corollary 5.5, $\left\{a^{R} / b^{R}\right\} \cup \mathbf{f}^{R}$ is a compatible set of quotients. Obviously $\left\langle\mathbf{f}^{R}\right\rangle \subseteq\left\langle\mathrm{f}^{R} \cup\left\{a^{R} / b^{R}\right\}\right\rangle$. As $\mathbf{f}^{*} \subseteq Q\left(\left\langle\mathbf{f}^{R}\right\rangle\right)$, this implies that $\left\{a^{R} / b^{R}\right\} \cup \mathbf{f}^{*}$ is compatible. Therefore, for all $c / d \in \mathbf{f}^{R} \cup \mathbf{f}^{*}, a \ominus b \leftrightarrow c \ominus d$. By Proposition 7.1, $a / b$ is covered by the test $\mathbf{f}^{R} \cup \mathbf{f}^{*}$. Since $a / b \perp \mathbf{f}^{R}, a / b$ is covered by $\mathbf{f}^{*}$.

Suppose that $a / b$ is covered by $\mathbf{f}^{*}$. As $\mathbf{f} \operatorname{loc} \mathbf{f}^{*}$, this implies that $a / b \lesssim \mathbf{f}^{*}$ and hence $a / b \perp \mathbf{f}$.

COROLLARY 9.2. Let $E$ be a complete lattice-ordered effect algebra. Let $\mathbf{f}, \mathbf{g}$ be events of $\Omega(E)$. Then $\mathbf{g} \lesssim \mathbf{f}$ if and only if every $a / b \in \mathbf{g}$ is covered by $\mathbf{f}^{* *}$.

Proof. Since $\mathbf{f} \operatorname{loc} \mathbf{f}^{*}, \mathbf{g} \lesssim \mathbf{f}$ if and only if $\mathbf{g} \perp \mathbf{f}^{*}$. By Proposition $6.8, \mathbf{g} \perp \mathbf{f}^{*}$ if and only if every $a / b \in \mathbf{g}$ is orthogonal to $\mathbf{f}^{*}$. By Proposition 9.1, $a / b \perp \mathbf{f}^{*}$ if and only if $a / b$ is covered by $\mathbf{f}^{* *}$.

Let us write, for $a / b \in Q(E)$ and $p \in S(E),(a / b) \sqcap p=a \wedge p / b \wedge p$. Note that the reduction map is a special case of $\Pi$ since $a^{R} / b^{R}=(a / b) \sqcap(a \ominus b)^{\uparrow}$.

LEMMA 9.3. Let $E$ be a complete lattice-ordered effect algebra and let $p, a, b \in E$. If $p \leftrightarrow a, b$ then $\{a / b\} \sim\left\{(a / b) \sqcap p,(a / b) \sqcap p^{\prime}\right\}$.

Proof. Let $L$ be a finite compatible 0,1-sublattice of $E$ with $a, b, p \in L$. An easy computation in $B(L)$ yields $(a \wedge p+b \wedge p) \dot{\vee}\left(a \wedge p^{\prime}+b \wedge p^{\prime}\right)=a+b$. By Proposition 8.3, $\{a / b\} \sim\left\{(a / b) \sqcap p,(a / b) \sqcap p^{\prime}\right\}$.

LEMMA 9.4. If $E$ is a complete lattice-ordered effect algebra then

$$
s \wedge(s \wedge t)^{\uparrow} \leftrightarrow t \wedge(s \wedge t)^{\uparrow} \text { for all } s, t \in E .
$$

ProOF. We have

$$
s \wedge t=s \wedge(s \wedge t) \leq s \wedge(s \wedge t)^{\uparrow} .
$$

Similarly, $s \wedge t \leq t \wedge(s \wedge t)^{\uparrow}$. Thus, we have

$$
s \wedge(s \wedge t)^{\uparrow}, t \wedge(s \wedge t)^{\uparrow} \in\left[s \wedge t,(s \wedge t)^{\uparrow}\right] .
$$

By Proposition 5.1, $\left[s \wedge t,(s \wedge t)^{\uparrow}\right]$ is a compatible set, hence

$$
s \wedge(s \wedge t)^{\uparrow} \leftrightarrow t \wedge(s \wedge t)^{\uparrow} .
$$

LEMMA 9.5. Let $E$ be a complete lattice ordered effect algebra. Let $a / b, c / d \in$ $Q(E)$ be reduced. Then $x / y \lesssim(a / b),(c / d)$ if and only if

$$
\begin{aligned}
& x / y \lesssim(a / b) \sqcap((a \ominus b) \wedge(c \ominus d))^{\uparrow} \quad \text { and } \\
& x / y \lesssim(c / d) \sqcap((a \ominus b) \wedge(c \ominus d))^{\uparrow} .
\end{aligned}
$$


PROOF. We may assume that $x / \dot{y}$ is reduced. Suppose that $x / y \lesssim(a / b),(c / d)$. Then $x \ominus y \leq(a \ominus b) \wedge(c \ominus d) \leq((a \ominus b) \wedge(c \ominus d))^{\uparrow}$. By Proposition 5.1, since $\{x \ominus y, a \ominus b,(a \ominus b) \wedge(c \ominus d)\}$ is a compatible set, $\left\{x \ominus y, a \ominus b,((a \ominus b) \wedge(c \ominus d))^{\uparrow}\right\}$ is a compatible set. As $x / y$ and $a / b$ are reduced quotients, Corollary 5.5 implies that $\left\{x, y, a, b,((a \ominus b) \wedge(c \ominus d))^{\uparrow}\right\}$ is a compatible set. By Lemma 9.3, we have

$$
\{x / y\} \lesssim\{a / b\} \sim\left\{(a / b) \sqcap((a \ominus b) \wedge(c \ominus d))^{\uparrow},(a / b) \sqcap((a \ominus b) \wedge(c \ominus d))^{\uparrow}\right\} .
$$

Let $\mathbf{u}$ be a local complement of

$$
\left\{(a / b) \sqcap((a \ominus b) \wedge(c \ominus d))^{\uparrow},(a / b) \sqcap((a \ominus b) \wedge(c \ominus d))^{\uparrow \prime}\right\} .
$$

Since $\left\{x, y, a, b,((a \ominus b) \wedge(c \ominus d))^{\uparrow}\right\}$ is a compatible set and $x / y \perp \mathbf{u}$, we have $x \ominus y \leftrightarrow e \ominus f$, for all

$$
e / f \in\left\{(a / b) \sqcap((a \ominus b) \wedge(c \ominus d))^{\uparrow},(a / b) \sqcap((a \ominus b) \wedge(c \ominus d))^{\dagger^{\prime}}\right\} \cup \mathbf{u} .
$$

Therefore, by Proposition 7.1, $x / y$ is covered by

$$
\left\{(a / b) \sqcap((a \ominus b) \wedge(c \ominus d))^{\uparrow},(a / b) \sqcap((a \ominus b) \wedge(c \ominus d))^{\uparrow \prime}\right\} \cup \mathbf{u} .
$$

However, $x / y \perp \mathbf{u}$ and, since $x \ominus y \leq((a \ominus b) \wedge(c \ominus d))^{\uparrow} \in S(E), x / y$ and $a / b \sqcap((a \ominus b) \wedge(c \ominus d))^{\uparrow \prime}$ are disjoint. Therefore, $x / y \lesssim(a / b) \sqcap((a \ominus b) \wedge(c \ominus d))^{\uparrow}$. Symmetrically, one can prove $x / y \lesssim(c / d) \sqcap((a \ominus b) \wedge(c \ominus d))^{\uparrow}$.

The converse implication follows by Lemma 9.3 .

LEMMA 9.6. Let $E$ be a complete lattice-ordered effect algebra. Suppose that $a / b, c / d \in Q(E)$ are such that $a \ominus b \leftrightarrow c \ominus d$. Then $[\{a / b\}]_{\sim} \wedge[\{c / d\}]_{\sim}$ exists in $O(E)$ and equals $\left[\left\{a^{R} \wedge c^{R} /\left(b^{R} \vee d^{R}\right) \wedge\left(a^{R} \vee c^{R}\right)\right\}\right]_{\sim}$.

PROOF. Suppose that $x / y \lesssim a / b, c / d$. Since $\{a \ominus b, c \ominus d, x \ominus y\}$ is mutually compatible, Corollary 5.5 implies that $\left\{x^{R} / y^{R}, a^{R} / b^{R}, c^{R} / d^{R}\right\}$ is a compatible set of quotients. Thus, there is a finite compatible sublattice $L$ of $E$ with $\left\{x^{R} / y^{R}, a^{R} / b^{R}, c^{R} / d^{R}\right\} \subseteq Q(L)$. By Proposition 8.3, $x^{R}+y^{R} \leq a^{R}+b^{R}, c^{R}+d^{R}$ in $B(L)$. A simple calculation in $\mathrm{B}(\mathrm{L})$ then yields

$$
\left(a^{R}+b^{R}\right) \wedge\left(c^{R}+d^{R}\right)=\left(a^{R} \wedge c^{R}\right)+\left(\left(b^{R} \vee d^{R}\right) \wedge\left(a^{R} \vee c^{R}\right)\right)
$$

hence we obtain

$$
x^{R}+y^{R} \leq\left(a^{R} \wedge c^{R}\right)+\left(\left(b^{R} \vee d^{R}\right) \wedge\left(a^{R} \vee c^{R}\right)\right) \leq a^{R}+b^{R}, c^{R}+d^{R} .
$$

Again, by Proposition 8.3, we obtain

$$
x^{R} / y^{R} \lesssim a^{R} \wedge c^{R} /\left(b^{R} \vee d^{R}\right) \wedge\left(a^{R} \vee c^{R}\right) \lesssim a^{R} / b^{R}, c^{R} / d^{R} .
$$


LEMMA 9.7. Let $E$ be a complete lattice-ordered effect algebra. For all $a / b, c / d \in$ $Q(E),[\{a / b\}]_{\sim} \wedge[\{c / d\}]_{\sim}$ exists in $O(E)$.

PROOF. We may assume that $a / b$ and $c / d$ are reduced. Following Lemma 9.5, $[\{a / b\}]_{\sim} \wedge[\{c / d\}]_{\sim}$ exists if and only if

$$
\left[\left\{(a / b) \sqcap((a \ominus b) \wedge(c \ominus d))^{\uparrow}\right\}\right]_{\sim} \wedge\left[\left\{(c / d) \sqcap((a \ominus b) \wedge(c \ominus d))^{\uparrow}\right\}\right]_{\sim}
$$

exists, and if one exists, and hence the other does too, then they are equal. Let $M$ be a block of $E$ with $a \ominus b,(a \ominus b) \wedge(c \ominus d) \in M$. By Proposition 5.1, $((a \ominus b) \wedge(c \ominus d))^{\uparrow} \in M$. Since $M \cap S(M)=C(M),((a \ominus b) \wedge(c \ominus d))^{\uparrow}$ is central in $M$. Therefore,

$$
\begin{aligned}
\mid(a / b) & \sqcap((a \ominus b) \wedge(c \ominus d))^{\uparrow} \mid \\
= & \left(a \wedge((a \ominus b) \wedge(c \ominus d))^{\uparrow}\right) \ominus\left(b \wedge((a \ominus b) \wedge(c \ominus d))^{\uparrow}\right) \\
= & (a \ominus b) \wedge((a \ominus b) \wedge(c \ominus d))^{\uparrow} .
\end{aligned}
$$

Similarly, we obtain

$$
\left|(c / d) \sqcap((a \ominus b) \wedge(c \ominus d))^{\uparrow}\right|=(c \ominus d) \wedge((a \ominus b) \wedge(c \ominus d))^{\uparrow} .
$$

By Lemma 9.4 (putting $s=a \ominus b$ and $t=c \ominus d$ ),

$$
(a \ominus b) \wedge((a \ominus b) \wedge(c \ominus d))^{\uparrow} \leftrightarrow(c \ominus d) \wedge((a \ominus b) \wedge(c \ominus d))^{\uparrow} .
$$

By Lemma 9.6,

$$
\left[\left\{(a / b) \sqcap((a \ominus b) \wedge(c \ominus d))^{\uparrow}\right\}\right]_{\sim} \wedge\left[\left\{(c / d) \sqcap((a \ominus b) \wedge(c \ominus d))^{\uparrow}\right\}\right]_{\sim}
$$

exists in $O(E)$.

THEOREM 9.8. Let $E$ be a complete lattice-ordered effect algebra. Then $O(E)$ is an orthomodular lattice.

PROOF. It is well known that an orthoalgebra is a lattice if and only if it is a (lower or upper) semilattice. Therefore, it suffices to prove that for every pair $\mathbf{f}, \mathbf{g}$ of events of $\Omega(E),[\mathbf{f}]_{\sim} \wedge[\mathbf{g}]_{\sim}$ exists in the orthoalgebra $O(E)$. Let us write

$$
\mathbf{f}^{* *}=\left\{a_{1} / b_{1}, \ldots, a_{n} / b_{n}\right\} \text { and } \mathbf{g}^{* *}=\left\{c_{1} / d_{1}, \ldots, c_{m} / d_{m}\right\} .
$$

For $i \in\{1, \ldots, n\}$ and $j \in\{1, \ldots, m\}$ let $e_{i j} / f_{i j}$ be such that

$$
\left[\left\{e_{i j} / f_{i j}\right\}\right]_{\sim}=\left[\left\{a_{i} / b_{i}\right\}\right]_{\sim} \wedge\left[\left\{c_{j} / d_{j}\right\}\right]_{\sim}
$$

and let

$$
\mathbf{h}=\left\{e_{i j} / f_{i j}: i \in\{1, \ldots, n\} \text { and } j \in\{1, \ldots, m\}\right\}
$$


By Theorem 4.7, $\mathbf{h}$ is an event of $\Omega(E)$ and, by Proposition $7.6, \mathbf{h} \lesssim \mathbf{f}^{* *}, \mathbf{g}^{* *}$. It remains to prove that, for every $\mathbf{u} \lesssim \mathbf{f}^{* *}, \mathbf{g}^{* *}$, we have $\mathbf{u} \lesssim \mathbf{h}$. Let $x / y \in \mathbf{u}$. By Corollary $9.2, x / y$ is covered by $\mathbf{f}^{* *}$ and $\mathbf{g}^{* *}$. By a simple induction with respect to $m$ and $n$, it is easy to prove that

$$
\{x / y\} \sim\left\{x_{i j} / y_{i j}: i \in\{1, \ldots, n\} \text { and } j \in\{1, \ldots, m\}\right\},
$$

where for each $x_{i j} / y_{i j}$ we have $x_{i j} / y_{i j} \sqsubseteq a_{i} / b_{i}, c_{j} / d_{j}$. This implies that, for all $i \in\{1, \ldots, n\}$ and $j \in\{1, \ldots, m\}$,

$$
\left[x_{i j} / y_{i j}\right]_{\sim} \leq\left[a_{i} / b_{i}\right]_{\sim} \wedge\left[c_{j} / d_{j}\right]_{\sim} .
$$

Consequently, by Proposition 7.6, $x / y \lesssim \mathbf{h}$ and, again by Proposition $7.6, \mathbf{u} \lesssim \mathbf{h}$.

\section{0. $\phi_{E}, \phi_{E}^{*}$, compatibility and blocks}

In this section, we shall show that there is one to one correspondence between blocks of a complete lattice-ordered effect algebra $E$ and blocks of $O(E)$. Under $\phi_{E}$, the (pre)image of a block is always a block. Moreover, we prove that $E$, as a lattice, embeds into $O(E)$.

LEMMA 10.1. Let $E$ be a complete lattice-ordered effect algebra and let $\mathbf{f}$ be $a$ reduced event of $\Omega(E)$. Then

$$
\mathbf{f} \sim\left\{|\mathbf{f}|^{\downarrow} / 0\right\} \cup\left\{(a / b) \sqcap|\mathbf{f}|^{\downarrow^{\prime}}: a / b \in \mathbf{f}\right\}
$$

ProOF. Since $\mathbf{f}$ is reduced, $\mathbf{f}$ is compatible. Let $M$ be a block with $\mathbf{f} \subseteq Q(M)$. Since $|\mathbf{f}| \in M,|\mathbf{f}|^{\downarrow} \in M$. Therefore, for all $a / b \in \mathbf{f}$ we have $|\mathbf{f}|^{\downarrow} \leftrightarrow a, b$. By Lemma 9.3,

$$
\{a / b\} \sim\left\{(a / b) \sqcap|\mathbf{f}|^{\downarrow},(a / b) \sqcap|\mathbf{f}|^{\downarrow}\right\}
$$

hence

$$
\mathbf{f} \sim \bigcup_{a / b \in \mathbf{f}}\left\{(a / b) \sqcap|\mathbf{f}|^{\downarrow},(a / b) \sqcap|\mathbf{f}|^{\downarrow \prime}\right\}
$$

Since $|\mathbf{f}|^{\uparrow} \in C(M)$, we have

$$
|\mathbf{f}|=\bigoplus_{a / b \in \mathbf{f}} a \ominus b=\bigoplus_{a / b \in \mathbf{f}}(a \ominus b) \wedge|\mathbf{f}|^{\downarrow} \bigoplus_{a / b \in \mathbf{f}}(a \ominus b) \wedge|\mathbf{f}|^{\prime \prime}
$$


Since $|\mathbf{f}|^{\downarrow} \leq|\mathbf{f}|$ and $|\mathbf{f}|^{\downarrow} \in S(E)$, we see that

$$
|\mathbf{f}|^{\downarrow}=\bigoplus_{a / b \in \mathbf{I}}(a \ominus b) \wedge|\mathbf{f}|^{\downarrow} .
$$

Since, for all $a / b \in \mathbf{f}$,

$$
\left.|(a / b) \sqcap| \mathbf{f}\right|^{\downarrow} \mid=\left(a \wedge|\mathbf{f}|^{\downarrow}\right) \ominus\left(b \wedge|\mathbf{f}|^{\downarrow}\right),
$$

we obtain

$$
|\mathbf{f}|^{\downarrow}=\bigoplus_{a / b \in \mathbf{l}}\left|\left\{(a / b) \sqcap|\mathbf{f}|^{\downarrow}\right\}\right|=\left|\left\{|\mathbf{f}|^{\downarrow} / 0\right\}\right| .
$$

By Proposition 8.1, this implies that

$$
\bigcup_{a / b \in \mathbf{f}}\left\{(a / b) \sqcap|\mathbf{f}|^{\downarrow}\right\} \sim\left\{|\mathbf{f}|^{\downarrow} / 0\right\} .
$$

The rest follows from (10.1).

LEMMA 10.2. Let $E$ be a complete lattice-ordered effect algebra and let $[\mathbf{f}]_{\sim},[\mathbf{g}]_{\sim} \in O(E)$. Then $[\mathbf{f}]_{\sim} \leftrightarrow \leftrightarrow_{(E)}[\mathbf{g}]_{\sim}$ if and only if $\phi_{E}\left([\mathbf{f}]_{\sim}\right) \leftrightarrow_{E} \phi_{E}\left([\mathbf{g}]_{\sim}\right)$.

PROOF. We may assume that $f$ and $g$ are reduced. By Lemma 10.1, we have

$$
\begin{aligned}
& \mathbf{f} \sim\left\{|\mathbf{f}|^{\downarrow} / 0\right\} \cup\left\{(a / b) \sqcap|\mathbf{f}|^{\downarrow \prime}: a / b \in \mathbf{f}\right\}^{R}, \\
& \mathbf{g} \sim\left\{|\mathbf{g}|^{\downarrow} / 0\right\} \cup\left\{(a / b) \sqcap|\mathbf{g}|^{\downarrow \prime}: a / b \in \mathbf{g}\right\}^{R} .
\end{aligned}
$$

Let $M$ be a block with $|\mathbf{f}|,|\mathbf{g}| \in M$. Then $\left[|\mathbf{f}|^{\downarrow},|\mathbf{f}|\right] \cup\left[|\mathbf{g}|^{\downarrow},|\mathbf{g}|\right] \in M$. Since, for all $c / d \in\left\{|\mathbf{f}|^{\downarrow} / 0\right\} \cup\left\{(a / b) \sqcap|\mathbf{f}|^{\downarrow^{\prime}}: a / b \in \mathbf{f}\right\}^{R} \cup\left\{|\mathbf{g}|^{\downarrow} / 0\right\} \cup\left\{(a / b) \sqcap|\mathbf{g}|^{\downarrow^{\prime}}: a / b \in \mathbf{g}\right\}^{R}$, $c \ominus d \in M$, we have $c, d \in M$ for all such $c / d$. Let $L$ be a finite compatible 0,1 -sublattice of $E$ such that

$$
\left\{|\mathbf{f}|^{\downarrow} / 0\right\} \cup\left\{(a / b) \sqcap|\mathbf{f}|^{\downarrow^{\prime}}: a / b \in \mathbf{f}\right\}^{R} \cup\left\{|\mathbf{g}|^{\downarrow} / 0\right\} \cup\left\{(a / b) \sqcap|\mathbf{g}|^{\downarrow^{\prime}}: a / b \in \mathbf{g}\right\}^{R} \subseteq Q(L) .
$$

Let $\mathbf{t}_{L}$ be the test $\left\{e / f: e \in J(L)\right.$ and $\left.e \succ_{L} f\right\}$.

By Proposition 8.3 and Corollary 6.7, it is easy to check that for every $c / d \in Q(L)$ there exists $\mathbf{h} \subseteq \mathbf{t}_{\mathbf{L}}$ such that $\mathbf{h} \sim\{c / d\}$. Therefore [f] $\sim$ and $[\mathbf{g}] \sim$ are covered by the word $\left([\{a / b\}]_{\sim}: a / b \in \mathbf{t}_{f}\right)$. Thus $[\mathbf{f}]_{\sim} \leftrightarrow[\mathbf{g}]_{\sim}$.

THEOREM 10.3. Let $E$ be a complete lattice-ordered effect algebra.

(a) If $M$ is a block of $E$ then $\phi_{E}^{-1}(M)$ is a block of $O(E)$.

(b) If $B$ is a block of $O(E)$ then $\phi_{E}(B)$ is a block of $E$. 
PROOF. (a) By Lemma $10.2, \phi_{E}^{-1}(M)$ is a compatible subset of $O(E)$. We shall prove that $\phi_{E}^{-1}(M)$ is a maximal compatible subset of $O(E)$. Let $y \in O(E)$ and suppose that, for all $x \in \phi_{E}^{-1}(M), x \leftrightarrow y$. Then, $\phi_{E}\left(\{y\} \cup \phi_{E}^{-1}(M)\right) \supseteq M$ is compatible in $E$ by Lemma 10.2. Since $M$ is a maximal compatible subset of $E$, $\phi_{E}\left(\{y\} \cup \phi_{E}^{-1}(M)\right)=M$. Therefore $\phi_{E}(y) \in M$ and $y \in \phi_{E}^{-1}(M)$.

(b) $\phi_{E}(B)$ is compatible. Let $M \supseteq \phi_{E}(B)$ be a block of $E$. By part (a), $\phi_{E}^{-1}(M)$ is a block of $O(E)$. By the maximality of $B, B=\phi_{E}^{-1}(M)$ and we see that $\phi_{E}(B)=\phi_{E}\left(\phi_{E}^{-1}(M)\right)=M$.

For a complete lattice-ordered effect algebra $E$, a mapping $\phi_{E}^{*}: E \rightarrow O(E)$ is defined by $\phi_{E}^{*}(x)=[\{x / 0\}]_{\sim}$. Note that $\phi_{E}\left(\phi_{E}^{*}(x)\right)=x$.

LEMMA 10.4. Let $E$ be a complete lattice-ordered effect algebra, let $a / b \in Q(E)$ be reduced and let $p \in S(E)$ be such that $p \leftrightarrow a \ominus b$. Then $(a / b) \sqcap p$ is reduced.

PROOF. We shall prove that $x \leq b \wedge p$ and $x \wedge((a \wedge p) \ominus(b \wedge p))=0$ imply that $x=0$.

Note that, since $a / b$ is reduced and $x, p \leftrightarrow a \ominus b,\{x, a, b, p\}$ is a compatible set; let $M \supseteq\{x, a, b, p\}$ be a block of $E$. We have $(a \wedge p) \ominus(b \wedge p)=(a \ominus b) \wedge p$ since $p$ is central in $M$. Moreover,

$$
\text { (10.2) } \begin{aligned}
x \wedge(a \ominus b) & =x \wedge\left(((a \ominus b) \wedge p) \vee\left((a \ominus b) \wedge p^{\prime}\right)\right) \\
& =(x \wedge(a \ominus b) \wedge p) \vee\left(x \wedge(a \ominus b) \wedge p^{\prime}\right)=x \wedge(a \ominus b) \wedge p^{\prime}
\end{aligned}
$$

and, since $x \leq p, x \wedge(a \ominus b) \wedge p^{\prime}=0$. Since $x \leq b, x \wedge(a \ominus b)=0$ and $a / b$ is reduced, $x=0$.

THEOREM 10.5. Let E be a complete lattice ordered effect algebra. Then $\phi_{E}^{*}$ is a injective 0, 1-lattice homomorphism.

PROOF. It is obvious that $\phi_{E}^{*}(0)=0_{O(E)}$ and that $\phi_{E}^{*}(1)=1_{O(E)}$ and that $\phi_{E}^{*}$ is injective. Let $a, c \in E$. By Lemma 9.5,

$$
\begin{aligned}
\phi_{E}^{*}(a) \wedge \phi_{E}^{*}(c) & =[\{a / 0\}]_{\sim} \wedge[\{c / 0\}]_{\sim}=\left[\{a / 0\} \sqcap(a \wedge c)^{\uparrow}\right]_{\sim} \wedge\left[\{c / 0\} \sqcap(a \wedge c)^{\uparrow} / 0\right] \\
& =\left[\left\{a \wedge(a \wedge c)^{\uparrow} / 0\right\}\right]_{\sim} \wedge\left[\left\{c \wedge(a \wedge c)^{\uparrow} / 0\right\}\right]_{\sim} .
\end{aligned}
$$

By Lemma 9.4, $a \wedge(a \wedge c)^{\uparrow} \leftrightarrow a \wedge(a \wedge c)^{\uparrow}$, hence we may apply Lemma 9.6 to obtain

$\left[\left\{a \wedge(a \wedge c)^{\uparrow} / 0\right\}\right]_{\sim} \wedge\left[\left\{c \wedge(a \wedge c)^{\uparrow}\right\}\right]_{\sim}=\left[\left\{a \wedge c \wedge(a \wedge c)^{\uparrow} / 0\right\}\right]_{\sim}=[\{a \wedge c / 0\}]_{\sim}$ 
It remains to prove that $\phi_{E}^{*}$ preserves joins; in other words, that

$$
[\{a / 0\}]_{\sim} \vee[\{c / 0\}]_{\sim}=[\{a \vee c / 0\}]_{\sim} .
$$

This is equivalent to $[\{1 / a\}]_{\sim} \wedge[\{1 / c\}]_{\sim}=[\{1 / a \vee c\}]_{\sim}$. We have

$$
(1 / a)^{R}=a^{\prime \uparrow} / a \wedge a^{\prime \uparrow}, \quad(1 / c)^{R}=c^{\prime \uparrow} / c \wedge c^{\prime \uparrow} .
$$

By Lemma 9.5,

$$
\begin{aligned}
& {\left[\left\{a^{\prime \uparrow} / a \wedge a^{\prime \uparrow}\right\}\right]_{\sim} \wedge\left[\left\{c^{\prime \uparrow} / c \wedge c^{\prime \uparrow}\right\}\right]_{\sim}} \\
& \quad=\left[\left\{\left(a^{\uparrow} / a \wedge a^{\prime \uparrow}\right) \sqcap\left(a^{\prime} \wedge c^{\prime}\right)^{\uparrow}\right\}\right]_{\sim} \wedge\left[\left\{\left(c^{\uparrow} / c \wedge c^{\prime \uparrow}\right) \sqcap\left(a^{\prime} \wedge c^{\prime}\right)^{\uparrow}\right\}\right]_{\sim} .
\end{aligned}
$$

We see that

$$
\begin{aligned}
& \left(a^{\prime \uparrow} / a \wedge a^{\prime \uparrow}\right) \sqcap\left(a^{\prime} \wedge c^{\prime}\right)^{\uparrow}=\left(a^{\prime} \wedge c^{\prime}\right)^{\uparrow} / a \wedge\left(a^{\prime} \wedge c^{\prime}\right)^{\uparrow} \\
& \left(c^{\prime \uparrow} / c \wedge c^{\prime \uparrow}\right) \sqcap\left(a^{\prime} \wedge c^{\prime}\right)^{\uparrow}=\left(a^{\prime} \wedge c^{\prime}\right)^{\uparrow} / c \wedge\left(a^{\prime} \wedge c^{\prime}\right)^{\uparrow}
\end{aligned}
$$

and that, by Lemma 10.4 , both quotients are reduced. Moreover, since

$$
\left(a^{\prime} \wedge c^{\prime}\right)^{\uparrow} \ominus\left(a \wedge\left(a^{\prime} \wedge c^{\prime}\right)^{\uparrow}\right)=(1 \ominus a) \wedge\left(a^{\prime} \wedge c^{\prime}\right)^{\uparrow}=a^{\prime} \wedge\left(a^{\prime} \wedge c^{\prime}\right)^{\uparrow}
$$

and, similarly,

$$
\left(a^{\prime} \wedge c^{\prime}\right)^{\uparrow} \ominus\left(c \wedge\left(a^{\prime} \wedge c^{\prime}\right)^{\uparrow}\right)=c^{\prime} \wedge\left(a^{\prime} \wedge c^{\prime}\right)^{\uparrow}
$$

Lemma 9.4 implies that they are compatible. Therefore, we may apply Lemma 9.6 to compute the meet of their perspectivity classes. After an easy computation we obtain

$$
\begin{gathered}
{\left[\left(a^{\prime} \wedge c^{\prime}\right)^{\uparrow} / a \wedge\left(a^{\prime} \wedge c^{\prime}\right)^{\dagger}\right]_{\sim} \wedge\left[\left(a^{\prime} \wedge c^{\prime}\right)^{\uparrow} / c \wedge\left(a^{\prime} \wedge c^{\prime}\right)^{\uparrow}\right]_{\sim}} \\
=\left[\left(a^{\prime} \wedge c^{\prime}\right)^{\uparrow} /(a \vee c) \wedge\left(a^{\prime} \wedge c^{\prime}\right)^{\dagger}\right]_{\sim} .
\end{gathered}
$$

Finally, it remains to observe that

$$
(1 / a \vee c)^{R}=\left(a^{\prime} \wedge c^{\prime}\right)^{\uparrow} /(a \vee c) \wedge\left(a^{\prime} \wedge c^{\prime}\right)^{\uparrow}
$$

COROLlaRY 10.6. Let $E$ be a complete lattice-ordered effect algebra. Then $\phi_{E}^{*}(S(E))$ is a sub-orthomodular lattice of $O(E)$.

PROOF. By Theorem 10.5, $\phi_{E}^{*}(S(E))$ is closed with respect to $0,1, \vee$, and $\wedge$. It remains to prove that $\phi_{E}^{*}(S(E))$ is closed with respect to'. Let $a \in S(E)$. Then $\phi_{E}^{*}(a)=[\{a / 0\}]_{\sim}$. In $O(E)$, we have $[\{a / 0\}]_{\sim}=[\{1 / a\}]_{\sim}$. Since $a, a^{\prime} \in S(E)$, $\{1 / a\}$ is a sharp event of $\Omega(E)$. By Proposition 8.1, $|\{1 / a\}|=\left|\left\{a^{\prime} / 0\right\}\right|$ implies that $\{1 / a\} \sim\left\{a^{\prime} / 0\right\}$ and we see that

$$
[\{1 / a\}]_{\sim}=\left[\left\{a^{\prime} / 0\right\}\right]_{\sim}=\phi_{E}^{*}\left(a^{\prime}\right) \in \phi_{E}^{*}(S(E)) .
$$




\section{References}

[1] M. K. Bennett and D. J. Foulis, 'Phi-symmetric effect algebras', Found. Phys. 25 (1995), 16991722.

[2] — , 'Interval and scale effect algebras', Adv. in Appl. Math. 19 (1997), 200-215.

[3] G. Cattaneo, 'A unified framework for the algebra of unsharp quantum mechanics', Internat. $J$. Theoret. Phys. 36 (1997), 3085-3117.

[4] C. C. Chang, 'Algebraic analysis of many-valued logics', Trans. Amer. Math. Soc. 89 (1959), 74-80.

[5] F. Chovanec and F. Kôpka, 'Boolean D-posets', Tatra Mt. Math. Publ 10 (1997), 1-15.

[6] A. Dvurečenskij and S. Pulmannova', 'Tensor product of D-posets and D-test spaces', Rep. Math. Phys. 34 (1994), 251-275.

[7] A. Dvurečenskij and S. Pulmannová, New Trends in Quantum Structures (Kluwer, Dordrecht and Ister Science, Bratislava, 2000).

[8] D. Foulis and M. K. Bennett, 'Tensor products of orthoalgebras', Order 10 (1993), 271-282.

[9] D. J. Foulis and M. K. Bennett, 'Effect algebras and unsharp quantum logics', Found. Phys. 24 (1994), 1325-1346.

[10] D. J. Foulis, R. Greechie and G. Rütimann, 'Filters and supports in orthoalgebras', Internat. J. Theoret. Phys. 35 (1995), 789-802.

[11] D. J. Foulis and C. H. Randall, 'Operational quantum statistics. I. Basic concepts', J. Math. Phys. 13 (1972), 1667-1675.

[12] R. Giuntini and H. Greuling, 'Toward a formal language for unsharp properties', Found. Phys. 19 (1989), 931-945.

[13] G. Grätzer, General Lattice Theory, second edition (Birkhäuser, 1998).

[14] R. Greechie, D. Foulis and S. Pulmannová, 'The center of an effect algebra', Order 12 (1995), 91-106.

[15] S. Gudder, 'S-dominating effect algebras', Internat. J. Theoret. Phys. 37 (1998), 915-923.

[16] —_, 'Sharply dominating effect algebras', Tatra Mt. Math. Publ. 15 (1998), 23-30.

[17] J. Hashimoto, 'Ideal theory for lattices', Math. Japon. 2 (1952), 149-186.

[18] G. Jenča, 'Blocks of homogeneous effect algebras', Bull. Austral. Math. Soc. 64 (2001), 81-98.

[19] _-, 'Finite homogeneous and lattice ordered effect algebras', Discrete Math. 272 (2003), 197-214.

[20] - 'Boolean algebras R-generated by MV-effect algebras', Fuzzy sets and systems 145 (2004), $279-285$.

[21] —_, 'Sharp and meager elements in orthocomplete homogeneous effect algebras', Technical report, (FEI STU Bratislava, 2004).

[22] G. Jenča and S. Pulmannová, 'Quotients of partial abelian monoids and the Riesz decomposition property', Algebra Universalis 47 (2002), 443-477.

[23] —_, 'Orthocomplete effect algebras', Proc. Amer. Math. Soc. 131 (2003), 2663-2671.

[24] G. Jenča and Z. Riečanová, 'On sharp elements in lattice ordered effect algebras', BUSEFAL 80 (1999), 24-29.

[25] F. Kôpka, 'D-posets of fuzzy sets', Tatra Mt. Math. Publ. 1 (1992), 83-87.

[26] F. Kôpka and F. Chovanec, 'D-posets', Math. Slovaca 44 (1994), 21-34.

[27] H. M. MacNeille, 'Extension of a distributive lattice to a Boolean ring', Bull. Amer. Math. Soc. 45 (1939), 452-455.

[28] D. Mundici, 'Interpretation of AF C*-algebras in Lukasziewicz sentential calculus', J. Funct. Anal. 65 (1986), 15-63.

[29] C. H. Randall and D. J. Foulis, 'Operational quantum statistics. II. Manual of operations and their logics', J. Math. Phys. 13 (1972), 1667-1675. 
[30] Z. Riečanová, 'A generalization of blocks for lattice effect algebras', Internat. J. Theoret. Phys. 39 (2000), 855-865.

[31] — 'Continuous lattice effect algebras admitting order continuous states', Fuzzy sets and systems 136 (2003), 41-54.

\section{Department of Mathematics}

Faculty of Electrical Engineering and Information Technology

Ilkovičova 3

81219 Bratislava

Slovakia

e-mail: gejza.jenca@stuba.sk 